\title{
Fabaceae del distrito inferior de las Yungas (Selva Pedemontana) de la provincia de Jujuy (Argentina)
}

\author{
FABACEAE FROM THE LOWER Yungas district (LOWLAND MOUNTAIN \\ FOREST) OF JUJUY PROVINCE (ARgENTINA)
}

\author{
Ana V. Carranza*(D), Luis H. Armella® y Álvaro E. Zenón $(\mathbb{D}$
}

\begin{abstract}
Cátedra de Botánica General, Herbario JUA, Facultad de Ciencias Agrarias, Universidad Nacional de Jujuy, Argentina.
\end{abstract}

*anavcarranza@fca.unju.edu.ar

Citar este artículo CARRANZA, A. V., L. H. ARMELLA \& Á. E. ZENÓN. 2020. Fabaceae del distrito inferior de las Yungas (Selva Pedemontana) de la provincia de Jujuy (Argentina). Bol. Soc. Argent. Bot. 55: 587-604.

DOI: https://doi. org/10.31055/1851.2372.v55. n4.27705

Recibido: 19 Mar. 2020

Aceptado: 29 Oct. 2020

Publicado en línea: 13 Nov. 2020

Publicado impreso: 20 Dic. 2020

Editor: Ramiro Aguilar (D)

ISSN versión impresa 0373-580X ISSN versión on-line 1851-2372

\section{SUMMARY}

Background and aims: Lowland Mountain Forest is the Yungas district of Argentina most affected by anthropic activities, with Calilegua National Park (Jujuy) being the largest protected area of this forest in Argentina. Fabaceae is one of the main families of Spermatophytes due to its diversity and economic importance and it is also one of the most important families of the Lowland Mountain Forest, because of its number of species and abundance of individuals, and the variety of growth habits. In the Flora of Jujuy, Fabaceae has not been approached. The aim of the study is to present a synthesis of the Fabaceae species from the Lowland Mountain Forest, as a contribution to the knowledge of this family in the province.

M\&M: We collected and identified specimens in reproductive state from the Lowland Mountain Forest of the Calilegua National Park. We also revised the collection of the JUA Herbarium and family's bibliography, recording the species present in Jujuy's Lowland Mountain Forest (Ledesma, Santa Barbara and San Pedro departments), and Flora del Cono Sur (http://www.darwin.edu.ar/proyectos/floraargentina/fa.htm), Documentae Florae Australis (https://www.darwin.edu.ar/iris) and Tropicos databases (https://www.tropicos.org/home).

Results and Conclusions: We registered 48 genera and 92 species, 47 of which are in the Calilegua National Park. Centrosema variifolium was collected for the first time for Jujuy and second for Argentina. Sesbania exasperata mentioned as a novelty for Jujuy. The distribution of Vachellia albicorticata and Senna obtusifolia is extended. Many of the species are in a state of conservation concern, due to clearing and logging activities.

\section{KEY WORDS}

Biodiversity, Calilegua National Park, Fabaceae, Jujuy, Lowland Mountain Forest, Yungas.

\section{RESUMEN}

Introducción y objetivo: La Selva Pedemontana es el distrito de las Yungas de Argentina más afectado por actividades antrópicas, siendo el Parque Nacional Calilegua (PN, Jujuy) el área de mayor superficie protegida de esta selva en Argentina. Fabaceae es una de las principales familias de Espermatófitas por su diversidad e importancia económica y también una de las más importantes de la Selva Pedemontana, por el número de especies, abundancia de individuos y variedad de hábitos. En la Flora de Jujuy esta familia no fue abordada. El objetivo del trabajo es presentar una síntesis de las especies de Fabaceae encontradas en Selva Pedemontana de Jujuy, como contribución al conocimiento de esta familia en la provincia.

M\&M: Se coleccionaron e identificaron ejemplares en estado reproductivo del área con Selva Pedemontana del PN Calilegua. Se revisó además la colección de ejemplares del Herbario JUA y bibliografía de la familia, registrando las especies presentes en Selva Pedemontana de Jujuy (departamentos Ledesma, Santa Bárbara y San Pedro), y las bases de datos Documentae Florae Australis (https://www.darwin.edu.ar/iris), Flora del Cono Sur (http://www.darwin.edu.ar/proyectos/floraargentina/fa.htm) y Tropicos (https://www.tropicos.org/home).

Resultados y Conclusiones: Se registraron 48 géneros y 92 especies, 47 de ellas están presentes en el PN Calilegua. Se coleccionó por primera vez para Jujuy y segunda para Argentina a Centrosema variffolium. Se menciona como novedad para Jujuy a Sesbania exasperata y amplía la distribución de Vachellia albicorticata y Senna obtusifolia. Muchas de las especies se encuentran en estado de conservación preocupante, por los desmontes y actividades de aprovechamiento forestal.

\section{Palabras clave}

Biodiversidad, Fabaceae, Jujuy, Parque Nacional Calilegua, Selva Pedemontana, Yungas. 


\section{INTRODUCCIÓN}

La Selva de Transición (Cabrera, 1976) también llamada Selva Pedemontana (Brown \& Ramadori, 1988) es el distrito inferior de las Yungas de Argentina y se desarrolla entre los 350 y 900 msnm aproximadamente, entremezclándose con el Bosque Chaqueño al Este y con la Selva Montana al Oeste. Es el distrito de las Yungas que más sufrió el impacto de la actividad humana (Castro, 1995; Oyarzabal et al., 2018), con fuerte reducción de la superficie que ocupaba y alteración de su diversidad y estructura a consecuencia de la implantación de cultivos como la caña de azúcar y cítricos entre otros, de la instalación de grandes ciudades (como San Miguel de Tucumán) y del aprovechamiento de sus maderas valiosas.

El Parque Nacional Calilegua (PN Calilegua), ubicado en la provincia de Jujuy, es el área protegida de Argentina con mayor superficie de Selva Pedemontana. Esta selva se encuentra mayormente en el Sector Este del Parque, y cubre unas 10.000 ha, que incluyen la zona de explotación petrolera Yacimiento Caimancito. La actividad de este yacimiento conlleva un impacto tanto por los derrames de crudo como por las tareas de mantenimiento de los caminos, ductos y pozos. La Selva Pedemontana en el PN Calilegua ocupa además pequeñas superficies de la zona baja de la Ruta Provincial no 83 (seccional Aguas Negras) y del Sector Sur, en los bordes del Río Colorado y del Arroyo Aguas Negras Sur (APN, 2009).

En Jujuy, también existen relictos de esta selva en zonas no protegidas de los departamentos San Pedro, Ledesma y Santa Bárbara. Generalmente se encuentran en bordes de ríos y de fincas dedicadas a plantaciones de caña de azúcar, cítricos y hortalizas ubicados en el valle del Río San Francisco, en zonas bajas próximas a la Serranía de Santa Bárbara, en las áreas de Santa Clara, Palma Sola, las cuencas de los Arroyos Santa Rita y Totorilla (zonas de Vinalito y El Talar).

Fabaceae es una de las principales familias de Espermatófitas tanto por su diversidad como por su importancia económica. Está representada en Argentina por unos 184 géneros y 1400 especies (Anton \& Zuloaga, 2019). Durante el estudio de las comunidades vegetales del PN Calilegua se observó que constituye una de las familias más importantes de la Selva Pedemontana, por el número de especies, abundancia de individuos, variedad de hábitos y provisión de recursos para la fauna, entre otras razones.
En la Flora de la provincia de Jujuy esta familia no fue abordada. El objetivo de este trabajo es presentar una síntesis de las especies de Fabaceae encontradas en la Selva Pedemontana del PN Calilegua durante el relevamiento de su vegetación como así también las registradas en el área correspondiente a este distrito en el resto de la provincia de Jujuy, como contribución al conocimiento y valoración de esta familia en la biodiversidad de las Yungas.

\section{Materiales Y MÉtodos}

Como parte del estudio de las comunidades vegetales del PN Calilegua, se realizaron recorridos de inspección y censos de vegetación durante el período 2006-2020. Se coleccionaron ejemplares de especies de Fabaceae en estado reproductivo, los que fueron identificados mediante bibliografía específica. Los especímenes se depositaron en el Herbario de la Facultad de Ciencias Agrarias, de la Universidad Nacional de Jujuy (JUA).

Se buscaron además en la colección del Herbario JUA las especies colectadas en el área de Selva Pedemontana de Jujuy: en los departamentos San Pedro, Ledesma y Santa Bárbara, entre los 350 y los 900 msnm (Fig. 1, en base a Morello et al., 2012). Para el género Erythrina se revisaron además las colecciones de los Herbarios MCNS y LIL. Se excluyeron las especies que crecen sólo cultivadas, tanto para arbolado público u ornamental como para cortinas forestales o con fines agropecuarios.

Sobre la base de las especies censadas y coleccionadas en el Herbario JUA se elaboró una clave artificial, dando prioridad a caracteres que faciliten la identificación a campo: formas de crecimiento, tipo de hojas, presencia o ausencia de espinas o aguijones, tipos de flores y frutos, pubescencia, corteza en caso de árboles, lo cual varía según los dilemas. El material examinado se detalla en el Anexo 1.

Para las flores se considera: "flores papilionadas": corresponden a las flores de la subfamilia Papilionoideae; "flores mimosoides": corresponden a las flores del clado Mimosoide (ex-subfamilia Mimosoideae) (LPWG, 2017).

Se constató en la bibliografía de la familia la presencia en el área de estudio de otras especies no coleccionadas en el Herbario JUA, incluyendo para el análisis del número total de especies y géneros, 
A. V. Carranza et al. - Fabaceae de Selva Pedemontana de Jujuy

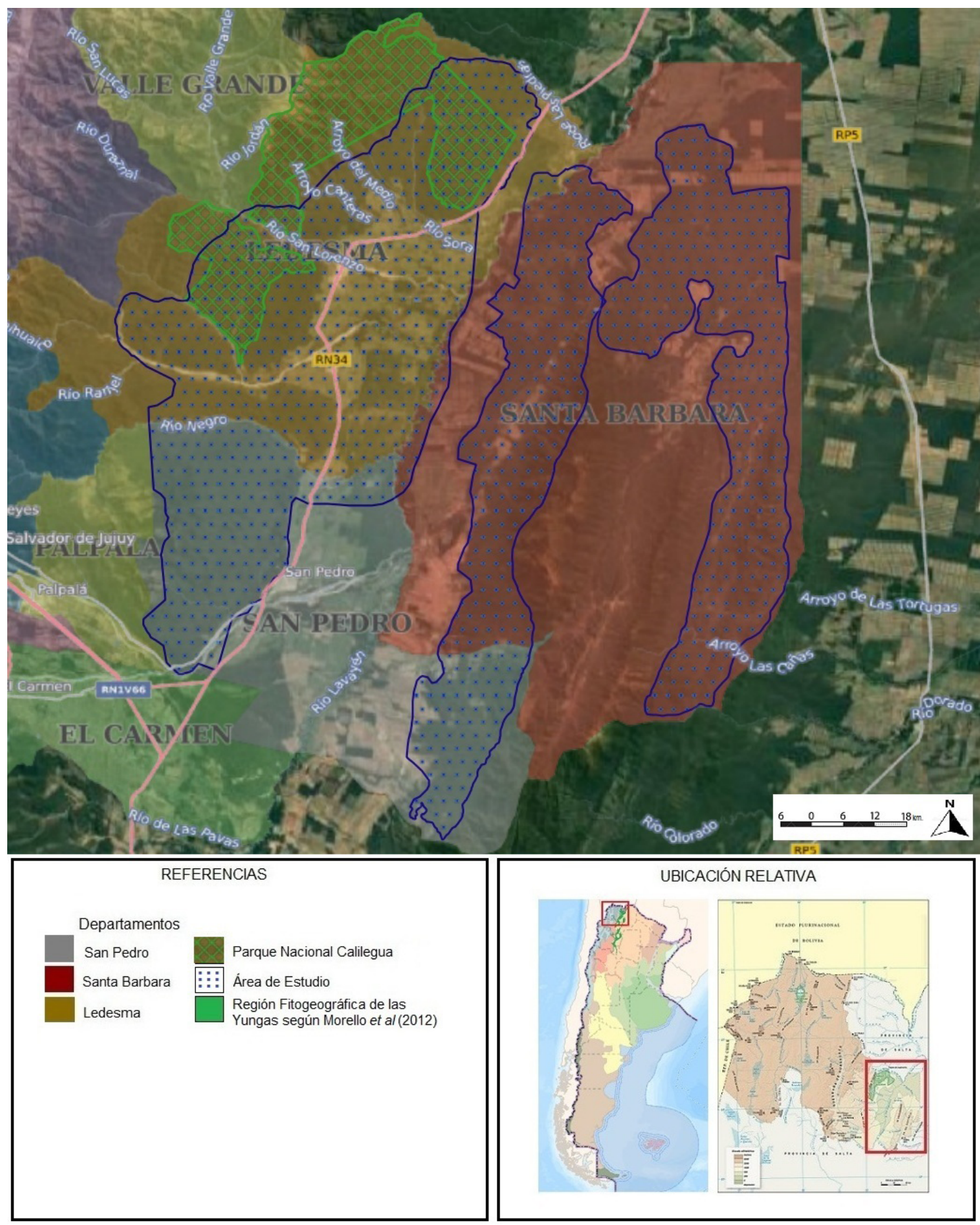

Fig. 1. Ubicación del área de estudio. 
distribución y estado de conservación aquellas cuyos ejemplares de referencia están incluidos en esta área. Estas especies y sus ejemplares de referencia se detallan en el Anexo 2.

Para corroborar datos de distribución en Argentina y en la provincia de Jujuy, se consultaron, además de la bibliografía de referencia, las bases de datos Documentae Florae Australis (2020) (https://www. darwin.edu.ar/iris), Flora del Cono Sur (Zuloaga \& Belgrano, 2020) (http://www.darwin.edu.ar/ proyectos/floraargentina/fa.htm) y Tropicos (2020) (https://www.tropicos.org/home). Para los nombres de las especies se siguió a Zuloaga \& Belgrano (2020).

Para las especies introducidas (no nativas) se tienen en cuenta las categorías planteadas por Blackburn et al. (2011), consideradas bajo los siguientes nombres:

Especies adventicias (casual species): especies liberadas en el ambiente, cuyos individuos sobreviven o no durante un período significativo, los que sobreviven pueden o no reproducirse, pero las poblaciones no son autosustentables (categorías $\mathrm{C} 0, \mathrm{C} 1, \mathrm{C} 2$ ).

Especies naturalizadas (naturalized species): los individuos sobreviven en el ambiente en que fueron liberados un período significativo y se reproducen formando poblaciones autosustentables (categoría C3) y poblaciones autosustentables con individuos que sobreviven y se reproducen a una significativa distancia del punto de liberación (categorías D1 y D2).

Especies invasoras (invasive species): poblaciones autosustentables con individuos que sobreviven, se reproducen y dispersan en múltiples sitios en un gran espectro de hábitats y con ocurrencia en gran extensión (categoría E).

Para el estado de conservación se consultó la Lista Roja (UICN, 2020 https://www.iucnredlist.org/ assessment/red-list-index) y se analizó en función a los relevamientos de campo y a bibliografía de referencia.

\section{Resultados y Discusión}

Para el distrito de la Selva Pedemontana de la provincia de Jujuy se registró en el Herbario JUA un total de 34 géneros y 54 especies de Fabaceae, de las cuales 1 es endémica de Salta y Jujuy, 49 son nativas para Argentina, 3 son introducidas y 1 es de origen dudoso. En el PN Calilegua se encuentran 41 especies nativas, 1 especie endémica y 2 introducidas. Los ejemplares de especies no coleccionadas en el Herbario JUA que fueron reportadas para el área de estudio por otros autores suman otras 38 especies y 14 géneros; de ellas, 3 se encuentran en el PN Calilegua y otras 4 son introducidas para la Argentina. Esto implica un total de 92 especies y 48 géneros de Fabaceae en la Selva Pedemontana de Jujuy hasta el momento.

Se registraron por primera vez para Jujuy a Centrosema variifolium y a Sesbania exasperata. La primera de ellas fue descripta por Burkart (1937) sobre un ejemplar coleccionado en "Río Piedras, Orán, provincia de Salta" (Rodriguez 178 SI). El actual hallazgo constituye el segundo registro para el país y fue realizado en la zona sur del PN Calilegua, en el Arroyo Aguas Negras Sur (Carranza et al. 1636 JUA).

Sesbania exasperata está reportada para Chaco, Corrientes, Formosa, Salta, Tucumán y Santa $\mathrm{Fe}$ (Documentae Florae Australis, 2020; Zuloaga \& Belgrano, 2020; Tropicos, 2020). Nuestro ejemplar (Carranza et al. 3172 JUA) fue coleccionado en el Yacimiento Caimancito, en ambientes alterados alrededor de un pozo de petróleo activo.

Se amplía la distribución a la provincia de Jujuy para Vachellia albicorticata y Senna obtusifolia. Si bien ambas especies habían sido previamente coleccionadas en Jujuy, esta distribución no consta en Flora del Cono Sur (Zuloaga \& Belgrano, 2020). En el caso de Vachellia albicorticata, Cialdella (1984 y 1997) considera que en Argentina se encuentra sólo en Salta y Tucumán. Durante el presente estudio esta especie fue coleccionada en el Yacimiento Caimancito, dpto. Ledesma (Carranza et al. 1734 JUA) y en Vinalito, dpto. Santa Bárbara (Carranza et al. 4638 JUA). Asimismo, fueron observados varios ejemplares en la entrada a la localidad de Yuto, dpto. Ledesma (no coleccionados). En Documentae Florae Australis (2020) se menciona un ejemplar coleccionado, en la localidad de El Cucho, dpto. Dr. Manuel Belgrano (Deginani 17 SI). Se encontró también a Senna obtusifolia en el Yacimiento Caimancito, dpto. Ledesma (Carranza et al. $1853 \mathrm{JUA}$, Tolaba et al. 4945 JUA) y en Palma Sola, dpto. Santa Bárbara (Ahumada \& Castellón 4943 JUA). Esta especie había sido coleccionada anteriormente en el dpto. 


\section{A. V. Carranza et al. - Fabaceae de Selva Pedemontana de Jujuy}

Santa Bárbara, camino a Vinalito (Morrone 2894 SI) según consta en Documentae Florae Australis (2020) y Tropicos (2020).

Se registraron 7 especies introducidas en la provincia de Jujuy, 2 corresponden a la categoría "invasora" (E), 1 a "naturalizada" (C3 o D1) y 4 a la categoría "adventicia" ( $\mathrm{C} 0$ y $\mathrm{C} 1)$. Se considera a Melilotus albus "invasora" ya que se registró frecuentemente en censos realizados a unos $7 \mathrm{~km}$ dentro del PN Calilegua, en el Arroyo Sauzalito, como así también en los playones de los pozos petroleros y bordes de caminos y arroyos internos del Yacimiento Caimancito. También se la observó creciendo ( in coleccionar) en diferentes puntos a lo largo de la Ruta Nacional 34 en los departamentos Ledesma y San Pedro y en diversas localidades del dpto. Santa Bárbara (Palma Sola, El Piquete entre otras). Se la encuentra además en bordes de caminos, ríos y arroyos en otras localidades de Jujuy correspondientes a Selva Montana y Prepuna.

Otra especie "invasora" (E) es Leucaena leucocephala ssp. glabrata. Ulibarri (2004) la cita por primera vez como adventicia en Argentina ("asilvestrada"), en la provincia de Jujuy, y destaca que "en estos momentos estaríamos frente a la naturalización de la subespecie". Actualmente, $L$. leucocephala ssp. glabrata, crece espontáneamente en orillas de caminos y terrenos alterados, encontrándose en grandes parches a lo largo de la Ruta Nacional 34 en el dpto. San Pedro (entre San Pedro y El Cuarteadero, en proximidades a Arrayanal y en matorrales al Sur de El Quemado) y en el dpto. Ledesma (entre Calilegua y el acceso a Yuto). Recientemente fue registrada dentro del PN Calilegua en el Yacimiento Caimancito. Además, existen ejemplares espontáneos adyacentes a su cultivo en otras áreas de Jujuy.

Neonotonia wightii fue coleccionada en el dpto. Santa Bárbara (García 603 JUA, Ahumada \& Castellón 4686 JUA). En Documentae Florae Australis (2020) también consta su colección en el mismo departamento (Cabrera et al. 33987 SI, Slanis $50 \mathrm{SI})$. Se considera a esta especie "adventicia" (C1), aunque por su aspecto semejante a otras especies de trepadoras trifolioladas, podría encontrarse más difundida sin haber sido detectada y corresponder a la categoría "naturalizada" (C3).

Leptospron adenanthum fue reportada por Zalocchi et al. (1995) para el dpto. San Pedro (Palacios 1209 BAFC). Se considera a esta especie "naturalizada" para Jujuy (categoría C3 o D1) ya que en Documentae Florae Australis (2020) existen varios ejemplares en la provincia, en el dpto. Santa Bárbara (Cabrera \& Fabris 19906 SI), y en el dpto. San Pedro (Krapovickas et al. 18624 SI, Krapvickas \& Schinini 39302 SI, Venturi 5088 SI), siendo una especie naturalizada en una amplia zona de la Argentina.

Sesbania bispinosa, especie de origen africano, fue coleccionada sólo dos veces en Jujuy (Cabrera et al. 31366 SI y Rossow \& Fortunato 732 BAB) (Fortunato, 1984). Por ello, se considera a Sesbania bispinosa como "adventicia" para Jujuy, correspondiéndole la categoría $\mathrm{C} 0$. Su distribución en América está reportada para el Caribe (Tropicos, 2020), Guyana, Salta y Jujuy (Fortunato, 1984) y para Tucumán (Tropicos, 2020; Documentae Flora Australis, 2020).

Lo mismo se observa para Vigna luteola, que fue coleccionada una vez por Novara \& Subils 2745 (S, SI) (Hoc et al., 2006; Documentae Flora Australis, 2020), por lo que se la considera "adventicia" de la categoría C0 para Jujuy. Sin embargo, esta especie está citada en un amplio rango en Argentina (Selvas Marginales de las cuencas de los ríos Paraná y Uruguay, en las provincias de Formosa, Misiones, Corrientes, Entre Ríos, norte de Buenos Aires, Salta, Santiago del Estero y Córdoba, además de Jujuy (Hoc et al., 2006), por lo cual podría pasar a otra categoría según los manejos en las vías de introducción.

También Bauhinia forticata ssp. pruinosa se considera "adventicia" (C0), habiendo sido coleccionada por Spegazzini 13822 (BAB) en Ledesma (dpto. Ledesma) (Fortunato, 1986; Fortunato \& Ulibarri, 1997). Esta especie es nativa del Noreste de Argentina y otras zonas cercanas de Paraguay, Brasil y Uruguay y se encuentra naturalizada a partir de su cultivo como ornamental en el Norte y Centro de Argentina (Fortunato, 1986).

Respecto a Erythrina mulungu, Lozano \& Zapater (2010) la consideran nativa en Jujuy, en tanto que Burkart (1952) sostiene que es cultivada. En base al análisis de la distribución de la especie durante los recorridos de colección y de los ejemplares coleccionados (Herbarios JUA, MCNS y LIL y Documentae Florae Australis, 2020), no se pudo llegar a una conclusión fehaciente al respecto. Por ello, en el presente trabajo se considera de origen "dudoso" para Jujuy a esta especie. 
En relación a Caesalpinia pulcherrima, Boelcke (1946) la considera como "especie indígena (nativa) utilizada para cultivo" y cita un ejemplar de Burkart, el cual sólo indica "Jujuy, Ledesma" sin especificar si es cultivado o no. En Zuloaga \& Belgrano (2020) la especie está catalogada como "adventicia" para Brasil (Santa Catarina) y Paraguay (Cordillera), sin distribución en Argentina. Sin embargo Ulibarri (1996) la considera como "probablemente originaria de América" e indica, al igual que Boelcke (1946) y Burkart (1952), su característica de utilizada como especie ornamental. Por ello, en el presente trabajo se la consigna como "nativa".

El resto de las especies son nativas para Jujuy, de las cuales en Argentina 14 se distribuyen únicamente en Salta y Jujuy: Amburana cearensis, Arachis duranensis, Bauhinia mollis var. notophila, Chloroleucon chacöense, Ch. foliolosum, Desmodium bridgesii, D. glabrum, Inga edulis, I. saltensis, Lonchocarpus lilloi, Mimosa sensibilis var. sensibilis, Myroxylon peruiferum, Senegalia etilis y Stylosanthes scabra. Otras 5 especies en Argentina crecen sólo en Jujuy, Salta y Tucumán: Cascaronia astragalina, Coursetia brachyrhachis, Poissonia hypoleuca, Vachellia albicorticata y Vicia andicola y las variedades Mimosa polycarpa var. subandina y Senna pendula var. eriocarpa. Por su parte, Condylostylis candida está reportada sólo para Jujuy y Misiones (Hoc et al., 2006).

Respecto a Inga edulis, Zapater et al. (2014) sostienen que en el Noroeste de Argentina sólo existe Inga saltensis, encontrando que ejemplares antes considerados como Inga edulis var. parviflora Benth. corresponden a Inga saltensis. Sin embargo, los ejemplares identificados como Inga edulis var. parviflora: Lillo 5124 (LIL) (Hoc, 1990 y 2005) y Jorgensen 35911 (BAB) (Hoc, 1990) no fueron considerados por Zapater et al. (2014), por lo que Inga edulis var. parviflora se incluye para la zona de estudio en el Anexo 2 bajo su nombre actualmente aceptado (Inga edulis).

Lozano \& Zapater (2010) registraron un ejemplar de Erythrina falcata Benth. en un sitio donde existen numerosos ejemplares cultivados de distintas especies (Zapater 2777 MCNS, "ingreso al PN Calilegua, dpto. Ledesma, 2347'24,4" S $64^{\circ} 47^{\prime} 12,4^{\prime \prime}$ '"). Debido a que no se encontraron otros ejemplares en los herbarios consultados (JUA, MCNS y LIL) de esta especie en la zona de estudio ni en Selva Pedemontana de Salta ni de Tucumán, se considera que el ejemplar reportado por Lozano \& Zapater (2010) es cultivado y no se incluye la especie para el área de estudio.

Respecto a Prosopis alba, en el PN Calilegua se observó un ejemplar aislado, añoso, en el Yacimiento Caimancito $\left(23^{\circ} 39^{\prime} 8.10^{\prime}\right.$ S S, 643 $35^{\prime} 1.30^{\prime}$ W), probablemente cultivado, al igual que el especímen de la localidad de Palo Blanco (Carranza et al. 4499 JUA), ubicado en plena Selva Pedemontana. Sin embargo, esta especie se incluye para el área de estudio debido a que es una especie nativa chaqueña que crece abundantemente en áreas de Jujuy donde se entremezcla la Selva Pedemontana con el Chaco, como por ejemplo en la zona de La Esperanza, La Mendieta y alrededores de San Pedro (dpto. San Pedro), Chalicán (dpto. Ledesma) y Santa Clara, Palma Sola, Talar y Vinalito (dpto. Santa Bárbara). Todos los ejemplares coleccionados en el Herbario JUA corresponden a la variedad alba, no habiéndose registrado la var. panta. Esta última variedad está considerada para Jujuy (Burkart, 1976; Palacios \& Brizuela, 2005; Zuloaga \& Belgrano, 2020), pero no existen registros en las bases de datos (el ejemplar mencionado por Burkart (1976) está identificado como Prosopis alba var. alba (Carmelich $580 \mathrm{SI})$ ). Se considera que esta ausencia, al menos en el Herbario JUA, podría corresponder a la falta de colecciones y no a la inexistencia real en la provincia. Otras especies típicamente chaqueñas (como por ejemplo Prosopis nigra) fueron registradas en la zona de estudio por otros autores (Anexo 2), siendo muchas veces dificultosa la asignación de la correspondencia a uno u otro distrito (Chaco o Selva Pedemontana), debido al amplio ecotono que se registra en muchas áreas.

Martínez et al. (2013) consideran para Jujuy la presencia de Anadenanthera colubrina var. colubrina. Teniendo en cuenta los caracteres del fruto indicados por Altschul (1964) el ejemplar que Martínez et al. (2013) consideran como perteneciente a la var. colubrina (Ahumada \& Castellón 7282 JUA) corresponde a la var. cebil. Otros ejemplares depositados en el Herbario JUA pertenecen también a la var. cebil. Por ello, en la clave sólo se incluye esta variedad, sin embargo en el Anexo 2 se especifican los ejemplares de la var. colubrina del trabajo antes mencionado.

En su tesis doctoral, Drewes (1995) cita para Macroptilium lathyroides al ejemplar Vervoorst y 


\section{A. V. Carranza et al. - Fabaceae de Selva Pedemontana de Jujuy}

Bacígalupo 4667 para "JUJUY. Depto. Ledesma: Orán, ruta $34 \mathrm{Km}$ 2603”. Esta localización es errónea ya que no se corresponden provincia, con departamento ni localidad ya sea por el número de ruta (Ruta Nacional ${ }^{\circ} 34$ ) como por el kilometraje. El mismo error existe en la etiqueta del ejemplar del herbario del Instituto de Botánica Darwinion (SI), lo cual persiste en las otras citas (Drewes, 1997; Palacios et al., 2001). Por otra parte, Drewes (1995) presenta como material de referencia para "Salta, Dpto. Orán" el ejemplar Vervoorst 4667 (LIL, BAB), que aparentemente sería el mismo ejemplar depositado en otros herbarios. No existen en otras publicaciones menciones a M. lathyroides para Jujuy, si bien en Documentae Flora Australis (2020) figura un ejemplar para la zona de estudio (Spegazzini 15509 BAB: "Jujuy, Ledesma"), el cual no fue revisado en el presente trabajo. La especie se incluye por lo tanto en el Anexo 2 pero sería importante corroborar su presencia en la zona mediante otros estudios.

En relación al estado de conservación de las especies encontradas, en la Tabla 1 (material suplementario) se presentan las especies incluidas en la Lista Roja de UICN (2020), según la cual la única especie en mayor peligro es Amburana cearensis.
Otras 6 especies están en categoría VU (Chloroleucon chacöense, Coursetia brachyrhachis, Inga saltensis, Libidibia paraguariensis, Poissonia hypoleuca y Senegalia etilis), en tanto que Prosopis alba ( $\sin$ especificar variedad) está categorizado como NT y Prosopis nigra como DD. Todas ellas fueron evaluadas en enero de 1998. En la categoría LC figuran 28 especies, y el resto no están consideradas, como por ejemplo Centrosema variifolium y Gleditsia amorphoides var. amorphoides. Durante los últimos 20 años, en el área del Chaco y zonas próximas como es la Selva Pedemontana se produjo el avance de los desmontes para el cultivo de la soja (Volante et al., 2005; Gasparri \& Grau, 2009), con escasas o nulas acciones de conservación o restauración, lo que permite suponer un agravamiento en el estado de conservación de las especies.

Por otra parte, Myroxylon peruiferum, especie de alto valor maderero, fue categorizada recientemente como LC, pero Sartori et al. (2015) la ubican en la categoría NT, por el impacto de los desmontes y del aprovechamiento forestal. Durante el presente estudio en la Selva Pedemontana de Jujuy se registraron escasos ejemplares adultos por lo que se considera que esta especie debería categorizarse al menos como NT.

\section{Clave Artificial}

( ${ }^{\circ}$ : especies presentes en el PN Calilegua, *: especies introducidas, d: origen dudoso)

1. Árboles o arbustos.

2 Hojas uni o trifolioladas. Flores papilionadas.

3. Hojas unifolioladas; folíolo orbicular de 2-10 cm long., discolor con hipofilo lanoso. Flores celestes-azules vistosas, de 14-22 mm long., en racimos péndulos cortos. Legumbre indehiscente moniliforme, castaño oscuro, péndula, de (3-)5-9,5 cm long. Arbustos o arbolitos de 2-4 m alt.

Poissonia hypoleuca ${ }^{\circ}$

3'. Hojas trifolioladas.

4. Arbustos de 1-3 m alt., sin aguijones.

5. Arbustos pubescentes con pelos hialinos, uncinulados y adpresos. Hojas pinnado-trifolioladas; folíolos ovados a elípticos, mucronados, el apical de 1,9-11,5 cm long., los laterales menores. Flores violáceas de $1 \mathrm{~cm}$ long., en racimos axilares o terminales. Lomento de artejos ovoides pajizos, artejo de 13-22 x 4,5-6 $\mathrm{mm}$.

Desmodium bridgesii ${ }^{\circ}$

5'. Arbustos con pubescencia amarillo broncínea, más densa en ramas terminales y fruto, pelos no unicinulados. Hojas digitado-trifolioladas; folíolos oblanceolados a elípticos, mucronados, emarginados o agudos, de 2-8 cm long. Flores amarillas de 1,5-2 cm long., en racimos terminales. Legumbre ovoide inflada, de 2-3 cm long.

Crotalaria micans ${ }^{\circ}$ 


\section{Bol. Soc. Argent. Bot. 55 (4) 2020}

4' Árboles de 6-15 m alt., con aguijones en tronco (escasos) y en raquis foliar.

6. Folíolos anchamente ovados a elípticos de hasta $9 \mathrm{~cm}$ lat.; estipelas glandulares en la inserción del par basal de folíolos dispuestas lateralmente y de forma globosa. Legumbre fusiforme casi recta de 8-20 cm long., 1 (2-3) seminada. Flores rosadas o color salmón, en grandes racimos. Árboles de 10-15m alt., áfilos en la antesis o con hojas jóvenes; ramas del año no arqueadas ni agrupadas en ápices de ramas del año anterior. Corteza pardo claro muy rugosa y corchosa con surcos longitudinales anchos y profundos de hasta $3 \mathrm{~cm}$.

Erythrina mulungu, seibo rosado ${ }^{\mathrm{d}}$

6' Folíolos ovados a elípticos más angostos, de hasta 5,5 cm lat.; estipelas glandulares del par basal de folíolos de disposición frontal y forma cónica. Legumbre cilíndrica y arqueada, de 11-35 cm long., 1-11 seminada. Flores rojas, solitarias o en pseudoracimos. Árboles de hasta $8 \mathrm{~m}$ alt., frondosos en la antesis; ramas del año característicamente arqueadas y agrupadas en los ápices de ramas del año anterior. Corteza pardo oscura rugosa, con surcos menos profundos.

Erythrina crista-galli var. crista-galli, seibo, seibo rojo

2'. Hojas pinnadas o bipinnadas. Flores papilionadas o no.

7. Árboles con grandes espinas ramificadas (dendromorfas) de hasta $30 \mathrm{~cm}$ long. en tronco, ramas con espinas trífidas o dendromorfas. Hojas pinnadas y bipinnadas en la misma planta, las bipinnadas con 2-4 yugas. Flores unisexuales y hermafroditas, blanco-verdosas de 6-7 mm long., cáliz de sépalos libres, corola no papilionada; flores estaminadas con 5-6(11) estambres libres. Legumbre indehiscente algo carnosa, falcada, comprimida lateralmente, negra lustrosa de 5-9 x 2-4 x 0,8-1,2 $\mathrm{cm}$.

Gleditsia amorphoides var. amorphoides, espina corona ${ }^{\circ}$

7'. Árboles o arbustos con o sin espinas o aguijones, si tienen espinas éstas no son dendromorfas. Plantas con todas las hojas pinnadas o todas bipinnadas. Flores y frutos distintos.

8. Arbustos o subarbustos, con aguijones infraestipulares, raramente infrapeciolares. Hojas sensitivas, bipinnadas uniyugadas, cada pinna con 3 folíolos grandes de 0,6-7,5 x 0,2-3,1 cm, ovado-elípticos y el folíolo inferior interno rudimentario o nulo. Flores mimosoides rosadas en cabezuelas. Craspedio glabro o raramente setoso en el replum.

Mimosa sensibilis var. sensibilis

8'. Árboles, arbustos o subarbustos con o sin aguijones. Hojas no sensitivas, pinnadas o bipinnadas. Flores mimosoides o de otro tipo, fruto nunca craspedio.

9. Hojas pinnadas. Plantas inermes (excepto Geoffroea decorticans).

10. Hojas paripinnadas. Flores papilionadas o no.

11. Hojas con raquis alado. Folíolos grandes de 4,5-17 cm long., elípticos, ovados u obovados, de ápice generalmente agudo, los inferiores gradualmente menores. Flores blancas mimosoides, en espigas. Legumbre indehiscente, gruesa, cilíndrica. Árboles.

12. Hojas ligeramente pubescentes, 3-6 yugadas, raquis alado de 3-13(-22) mm lat. Ramas del año, pecíolo, raquis foliar y pedúnculo pilosos. Espigas cónicas. Flores con cáliz de 5-11 mm long.; corola de 10-19 mm long.; estambres de hasta 45(-60) $\mathrm{mm}$ long. Legumbre ocráceo-tomentosa, con márgenes engrosados pero que no ocultan las caras.

Inga saltensis

12 '. Hojas glabras, 2-3 yugadas, raquis angostamente alado de 2-10 mm lat., ala dilatada sólo por debajo de los folíolos. Ramas del año y pedúnculo glabros a pubérulos, pecíolo y raquis foliar pubérulos. Espigas cilíndricas. Flores menores, cáliz de 1-2 $\mathrm{mm}$ long.; corola de 4-5 mm long.; estambres de hasta $14 \mathrm{~mm}$ long. Legumbre glabra amarilla en fresco y parda o castaña al secarse, con márgenes no engrosados.

Inga marginata ${ }^{\circ}$ 


\section{A. V. Carranza et al. - Fabaceae de Selva Pedemontana de Jujuy}

11'. Hojas con raquis no alado. Folíolos menores, ovados a oblanceolados, agudos $\mathrm{u}$ obtusos. Flores no mimosoides, en racimos o panojas. Legumbre plana o cilíndrica, dehiscente o subdehiscente. Subarbustos, arbustos o árboles.

13. Flores blanquecinas papilionadas; estambres diadelfos. Folíolos de 6-27(-38) mm long. Raquis foliar prolongado en mucrón. Legumbre comprimida lateralmente, generalmente recta, ápice rostrado curvo.

14. Legumbre no resupinada. Pedúnculo menor a $1 \mathrm{~cm}$ long. Flores blancas, de 13$16 \mathrm{~mm}$ long. Folíolos angostamente elípticos u oblanceolados, de 2,5-4(-11) mm lat.; estipelas generalmente ausentes. Hojas 8-22 yugadas. Tallos prontamente lignificados y engrosados, glabros, con lenticelas. Plantas áfilas en la antesis. Arbustos o arbolitos de 1,5-4(-5) $\mathrm{m}$ alt.

Coursetia brachyrhachis ${ }^{\circ}$

14'. Legumbre resupinada. Pedúnculo de 1-5 cm long. Flores blancas, blancoamarillentas o blanco-rosadas, de $8-12 \mathrm{~mm}$ long. Folíolos oval-elípticos de 5-11(-18) mm lat.; estipelas presentes. Hojas 6-14 yugadas. Tallos no lignificados, delgados con escasas lenticelas. Plantas foliosas en la antesis. Arbustos gráciles o subarbustos de 0,5-2 $\mathrm{m}$ alt.

Coursetia hassleri ${ }^{\circ}$

13'. Flores amarillas no papilionadas, estambres 10 , libres ( 3 estaminodios superiores y 7 estambres inferiores fértiles subiguales o en 3 grupos de diferente tamaño). Folíolos mayores. Raquis foliar no prolongado en mucrón (excepto en Senna spectabilis). Legumbres cilíndricas curvas o rectas, péndulas.

15. Árboles o arbustos pubescentes, erectos. Folíolos de ápice agudo o acuminado.

16. Arbustos o árboles de hasta 10(-20) m alt. Hojas 8-15 yugadas; folíolos ovado-lanceolados, acuminados. Panículas terminales piramidales, grandes y vistosas de hasta $15 \mathrm{~cm}$ long. Estambres fértiles subiguales. Legumbre cilíndrica subtorulosa, negruzca, de hasta $30 \mathrm{~cm}$ long. Pubescencia corta amarillenta en tallos jóvenes y hojas.

Senna spectabilis var. spectabilis, carnaval ${ }^{\circ}$

16' Arbustos de 0,5-2 m alt. Hojas 3-4 yugadas; folíolos elípticos u oblongolanceolados, agudos. Racimos axilares cortos. Estambres fértiles en grupos. Legumbre cilindroide amarillenta, de hasta $6 \mathrm{~cm}$ long. Pubescencia blanquecina, pilosula o tomentulosa en toda la planta y lanosa en hipofilo.

Senna morongii

15'. Arbustos glabros, apoyantes, de 1-3 m alt. Folíolos de ápice redondeado, obovados. Hojas 3-5 yugadas. Estambres fértiles en grupos. Legumbre glabra y lustrosa, cilindroide, marrón grisácea, de 7-8 cm long., con suturas inmersas.

Senna pendula var. eriocarpa ${ }^{\circ}$

10'. Hojas imparipinnadas. Flores papilionadas (en Amburana cearensis reducida al estandarte).

17. Árboles de hasta $10 \mathrm{~m}$ alt. de porte tortuoso o arbustos, con algunas ramas espinescentes. Corteza rugosa y gruesa con surcos longitudinales, marrón claro, que se desprende en placas longitudinales en ramas y troncos jóvenes y deja expuesta la zona interna de color verdoso brillante. Fruto drupa. Hojas generalmente fasciculadas, 2-4 yugadas; raquis menor a $5 \mathrm{~cm}$ long.; folíolos oblongos-elípticos de hasta 2,5 cm long., ápice obtuso o emarginado. Flores amarillo-anaranjadas. Racimos de raquis corto.

Geoffroea decorticans var. decorticans, chañar 
17'. Árboles mayores, hasta 20 o $35 \mathrm{~m}$ alt., de fuste generalmente recto y largo, sin espinas. Cortezas distintas. Fruto sámara o legumbre. Hojas no fasciculadas (excepto en Cascaronia astragalina, que a veces presenta hojas fasciculadas), raquis mayor a 6 $\mathrm{cm}$ long. Flores amarillas, blancas o azules-púrpuras. Racimos cortos o largos.

18. Flores blancas o azules-púrpuras. Hojas con menos de 10 folíolos, éstos alternos u opuestos. Folíolos grandes, mayores a $2,5 \mathrm{~cm}$ long.

19. Flores azul-violáceas o púrpuras de 1,2-1,4 cm long. Folíolos opuestos, glabros, 1-2 yugas (3-5 folíolos por hoja), de 3-9 cm long., oblongos, acuminados. Ramas jóvenes con lenticelas amarillentas. Legumbre plana, obovado-lanceolada, indehiscente o tardíamente dehiscente, de $4-15 \mathrm{~cm}$ long.

Lonchocarpus lilloi, quina blanca ${ }^{\circ}$

19'. Flores blancas de menos de $1 \mathrm{~cm}$ long. Folíolos alternos, de 2-6(-7) cm long. Ramas jóvenes sin lenticelas.

20. Folíolos oval-lanceolados a orbiculares, agudos, de borde ondulado, 3-11 por hoja con rayas y puntos translúcidos (canales secretores); epifilo glabro con escasos tricomas ferrugíneos y cortos en vena media, hipofilo escasamente pubescente con tricomas similares. Corteza pardo-grisácea, con surcos longitudinales poco profundos y delgados, que no se desprende en placas papiráceas. Racimos de $5-15 \mathrm{~cm}$ long. Corola bien desarrollada, estandarte anchamente ovado de 3,5-6 $\mathrm{mm}$ long, los otros pétalos lanceolados más pequeños, no soldados; estambres cortos con anteras de 2-3 mm long. Sámara con ala asimétrica, semilla de posición apical, de 5-7 cm long. Leño no aromático.

Myroxylon peruiferum, quina, quina colorada ${ }^{\circ}$

20' Folíolos orbiculares o elípticos, obtusos o emarginados, no ondulados, 7-12 por hoja, sin rayas ni puntos translúcidos; epifilo glabro, hipofilo con pubescencia blanquecina. Corteza castaño rojiza ligeramente rugosa, delgada, desprendiéndose en láminas papiráceas irregulares que dejan a la vista la superficie inferior más clara. Racimos de $2-4 \mathrm{~cm}$ long. Corola reducida al estandarte, de 7-10 mm long.; estambres largos con anteras menores a $1 \mathrm{~mm}$ long. Legumbre de contorno oblongo y base alada, porción apical globosa, dehiscencia apical, de 6-10 cm long. Leño aromático.

Amburana cearensis, roble ${ }^{\circ}$

18'. Flores amarillas. Hojas con más de 12 folíolos, éstos generalmente opuestos pero también alternos o subopuestos. Folíolos menores a $3 \mathrm{~cm}$ long.

21. Folíolos elípticos de 10-32 x 3-8 mm, glabros. Ramas, hojas, flores y fruto con numerosas glándulas sésiles redondeadas oscuras. Flores de $0,7-1 \mathrm{~cm}$ long. Legumbre indehiscente samaroide, oblongo-elíptica romboidal, muy plana, de 3-4 cm long. Corteza parda clara corchosa, con surcos longitudinales profundos amarillentos de hasta $30 \mathrm{~cm}$ long. y $3 \mathrm{~cm}$ prof., más visibles en ramas jóvenes.

Cascaronia astragalina, tipa amarilla ${ }^{\circ}$

21' Folíolos elípticos, ovales u oblongos mayores, de 18-62 x 8-25 mm, glabros o escasamente pubescentes. Plantas sin glándulas. Flores mayores, de 1,5-2,5 cm long. Sámara estipitada con porción seminífera globosa basal y ala falcada, de 5-7 $\mathrm{cm}$ long. Corteza parda, rugosa, con surcos longitudinales no amarillentos, más cortos y menos profundos. 


\section{A. V. Carranza et al. - Fabaceae de Selva Pedemontana de Jujuy}

9' Hojas bipinnadas. Plantas con o sin espinas o aguijones.

22. Plantas inermes.

23. Árboles de corteza muy lisa y delgada grisácea, que se desprende en placas ovales y dejan al descubierto la superficie inferior verdosa o blanquecina. Folíolos simétricos, elípticos, obtusos, de 4-6 mm long. Pinnas distanciadas de modo que los folíolos no llegan a superponerse ni tocarse por su ápice. Flores amarillas de 7-9 mm long. con pétalos libres, ligeramente zigomorfas, en racimos cortos. Legumbre indehiscente gruesa, de contorno elíptico, apiculada y con márgenes marcados, marrón oscuro casi negra, lustrosa, de 1,5-4 x 1-2 x 0,5-1 cm.

Libidibia paraguariensis, guayacán ${ }^{\circ}$

23'. Árboles o arbustos con corteza distinta, que no se desprende en placas. Folíolos asimétricos, agudos. Pinnas no distanciadas, de modo que los folíolos se superponen o tocan por el ápice. Flores mimosoides blanquecinas, en cabezuelas o espigas. Legumbres dehiscentes o no, en este caso de forma distinta.

24. Hojas 2-7 yugadas. Folíolos mayores a $7 \mathrm{~mm}$ long., falcados o ligeramente falcados, de ápice acuminado, glabros. Árboles o arbustos. Flores en cabezuelas. Legumbre dehiscente plana o legumbre indehiscente.

25. Árboles de hasta $30 \mathrm{~m}$ alt. Legumbre indehiscente, gruesa, negruzca, formando un círculo incompleto, de 5-7 x 3-4 x 1-1,5 cm. Folíolos gradualmente menores hacia el ápice, de 5-20 mm long. Hojas 4-5 yugadas. Cabezuelas reunidas en racimos axilares menores que las hojas.

Enterolobium contortisiliquum, pacará, oreja de negro ${ }^{\circ}$

25'. Arbustos o árboles de menor tamaño. Legumbre plana y recta, de ápice redondeado con apículo. Folíolos gradualmente mayores hacia el ápice o de igual tamaño. Cabezuelas fasciculadas o en panojas.

26. Arbustos gráciles, de hasta $3 \mathrm{~m}$ alt. Hojas 2-4 yugadas, sin glándulas en pecíolo ni raquis. Folíolos elíptico-obovados, gradualmente mayores hacia el ápice, de 7-18 mm long. Cabezuelas 1-5, fasciculadas en axilas de ramas del año. Legumbre pardo clara, de 8-12 x 0,6-1 cm., dehiscencia apical elástica.

Zapoteca formosa ${ }^{\circ}$

26 '. Arbustos robustos o árboles de hasta 8(-15) m alt. Hojas 4-6(-9) yugadas, con glándulas sésiles en pecíolo y raquis. Folíolos elípticos, el primer par de $7 \mathrm{~mm}$ long., los restantes de subiguales $18-20 \mathrm{~mm}$ long. Cabezuelas reunidas en grandes panojas foliosas. Legumbre castaño-rojiza, de 10-20 x 1,5-2,0 cm., dehiscencia lateral no elástica.

Leucaena leucocephala spp. glabrata*

24'. Hojas (3-)7-30 yugadas. Folíolos menores a $6 \mathrm{~mm}$ long., rectos o falcados, glabros o pubescentes. Árboles de 20-30 m alt. Flores en cabezuelas o espigas. Legumbre siempre plana.

27. Folíolos oblongo-lanceolados de 4-10 x 1-2 mm, pubescentes. Flores en espigas axilares péndulas. Legumbre de 13-18 x 0,8-1,4 cm., castaño-rojiza a negra, bordes no engrosados, ligeramente contraídos entre las semillas. Corteza ligeramente rugosa, pardo-grisácea, sin mamelones.

Parapiptadenia excelsa, cebil blanco, horco cebil ${ }^{\circ}$

27 . Folíolos muy pequeños, oblongo-lineares, de 2-4 x 0,5-0,8 mm, glabros. Flores en cabezuelas axilares. Legumbre de 15-26 x 1,5-2,5 cm, castaño-rojiza algo brillante, bordes engrosados, rectos o contraídos entre las semillas. Corteza 
rugosa castaña con mamelones oscuros característicos, a veces escasos; ramas y troncos más jóvenes con corteza gris-parda similar a Parapiptadenia excelsa.

Anadenanthera colubrina var. cebil, cebil colorado ${ }^{\circ}$

22'. Plantas con espinas o aguijones. Flores mimosoides blancas, blanco-amarillentas o amarillas.

28. Árboles de 6-12(-15) m alt., de porte tortuoso. Hojas con 1-4 yugas. Flores en cabezuelas globosas o espigas péndulas. Espinas axilares, geminadas o solitarias, no presentes en todas las hojas, a veces estípulas espinescentes.

29. Hojas con 2-4 yugas. Folíolos asimétricos obovado-elípticos de 3-6 mm lat. Flores blancas de 10-13 mm long., en cabezuelas. Legumbre carnosa espiralada formando 2 a 3 espiras enrolladas sobre sí mismas, pardo-rojiza, dehiscente tardíamente o indehiscente. Corteza rugosa, corchosa, pardo clara, con placas cuadrangulares irregulares.

Chloroleucon tenuiflorum, tatané

29'. Hojas con 1-2(-3) yugas. Folíolos simétricos, oblongo-lanceolados, de 1,5-2,5 mm lat. Flores blanco-amarillentas de 8-9 mm long., en espigas péndulas. Legumbre indehiscente poco o muy carnosa, amarilla, ligeramente falcada a semicircular nunca espiralada. Corteza rugosa pardo oscura, no corchosa, con surcos longitudinales profundos, las capas superiores arqueadas y separándose del tronco en los extremos.

Prosopis alba var. alba, algarrobo blanco ${ }^{\circ}$

28'. Árboles pequeños de menos de $6 \mathrm{~m}$ alt. o arbustos. Hojas con (1-)3 o más yugas. Flores blancas o amarillas en cabezuelas globosas. Plantas con espinas estipulares o con aguijones.

30. Arbustos o arbustos apoyantes con aguijones dispersos. Flores blancas o blanco amarillentas. Legumbre, plana y recta. Hojas con 7-12 yugas.

31. Folíolos de 4-6 x 0,5-1 mm. Cabezuelas de 7-10 mm diám. Pecíolo con una glándula discoide o elipsoide, sésil, en porción media. Ramas y raquis tomentosos.

Senegalia parviceps, garabato ${ }^{\circ}$

31'. Folíolos mayores, de 5-9 x 1-2 mm. Cabezuelas de 15-20 mm diám. Pecíolo con una glándula estipitada cilíndrica en mitad distal del pecíolo. Ramas glabras, raquis pubescente.

Senegalia tucumanensis ${ }^{\circ}$

30'. Árboles o arbustos no apoyantes, con espinas estipulares. Flores amarillas. Legumbre indehiscente, cilíndrica o moniliforme. Hojas con (1-)3 o más yugas.

32. Árboles de $3-7(-8) \mathrm{m}$ alt. Corteza de hasta $3 \mathrm{~cm}$ prof., papirácea blanquecina en las capas profundas, tornándose amarillenta y grisácea hacia la superficie, que se desprende en numerosas láminas delgadas. Hojas con (1)3-4 yugas. Folíolos de 4-13 mm long. Legumbre indehiscente cilíndrica, recta o curva, de 4-10 cm long.

Vachellia albicorticata, tusca blanca ${ }^{\circ}$

32'. Árboles de menor altura o arbustos. Corteza rugosa pardo oscura, no papirácea, con surcos longitudinales delgados y angostos, arqueados en los extremos sin llegar a desprenderse. Hojas habitualmente con más de 4 yugas. Folíolos menores, de 1-4 mm long. Legumbre indehiscente comprimida, moniliforme, de 6-18 cm long. 


\section{A. V. Carranza et al. - Fabaceae de Selva Pedemontana de Jujuy}

33. Espinas cónicas, de 5-15(-30) mm long. Hojas con 10-25 yugas.

Vachellia aroma, tusca ${ }^{\circ}$

33'. Espinas comprimidas, de sección rómbica con costilla longitudinal, de 5-45(55) mm long. Hojas con 3-30 yugas.

Vachellia macracantha, tusca ${ }^{\circ}$

1' Hierbas, subarbustos o trepadoras herbáceas o leñosas.

34. Hojas bipinnadas. Flores mimosoides en cabezuelas.

35. Hojas 1-yugadas, sensitivas. Subarbustos con aguijones. Flores rosadas, cabezuelas con numerosas flores. Fruto craspedio (a veces legumbre en Mimosa debilis var. debilis).

36. 14-30 pares de folíolos por pinna, cada folíolo de 7-18 x 1,5-3,7 mm. Aguijones infraestipulares, geminados, caducos. Craspedio con caras híspidas y pubescentes, replum con fuertes cerdas divergentes de $5 \mathrm{~mm}$ long.

Mimosa polycarpa var. subandina ${ }^{\circ}$

36'. 3 folíolos desarrollados por pinna y 1 folíolo inferior interno rudimentario o nulo, folíolos desarrollados de 0,6-6 cm long.

37. Subarbustos o arbustos con muy pocos aguijones, infraestipulares raro infrapeciolares. Folíolo inferior interno ausente o rudimentario menor a $5 \mathrm{~mm}$ long., folíolos desarrollados ovados a elípticos, glaucos. Craspedio glabro o sólo híspido en el replum.

Mimosa sensibilis var. sensibilis

37'. Subarbustos con aguijones abundantes, en tallos y pecíolo, raro inermes. Folíolo inferior interno presente, de 1-10 mm long., folíolos desarrollados obovados. Craspedio (a veces legumbre) glabro a híspido en el replum y/o en artejos o valvas, cuando híspido con largas cerdas de $1-5 \mathrm{~mm}$ long.

Mimosa debilis var. debilis ${ }^{\circ}$

$35 '$ '. Hojas 2-5 yugadas, no sensitivas. Subarbustos o hierbas inermes. Flores blancas, cabezuelas con menos de 25 flores. Legumbre comprimida, larga, recta o ligeramente arqueada.

38. Hierbas postradas de $10-40 \mathrm{~cm}$ alt. Legumbre rostrada o acuminada, ligera o fuertemente arqueada, de 1,5-5 cm long.

Desmanthus acuminatus

38'. Hierbas erectas o subarbustos de hasta 1,5 m alt. Legumbre de ápice obtuso a agudo, recta, a veces algo arqueada, de 3,5-9 cm long.

Desmanthus virgatus ${ }^{\circ}$

34'. Hojas unifolioladas, trifolioladas o pinnadas. Flores no mimosoides, en racimos o fascículos.

39. Trepadoras herbáceas o leñosas, o hierbas decumbentes (postradas) o rastreras. Flores papilionadas.

40. Hojas imparipinnadas biyugadas. Racimos cortos axilares (a veces panículas) y numerosas flores axilares fasciculadas. Flores amarillas de 8-10 mm long. Sámara péndula, estipitada, de 3-4 cm long., semillas en la mitad basal cilíndrica, ala apical. Trepadoras volubles.

Nissolia fruticosa var. guatemalensis ${ }^{\circ}$

40'. Hojas uni o trifolioladas (en Centrosema variifolium algunas hojas con 4 o 5 folíolos). Racimos axilares o terminales. Flores amarillas, blanco-verdosas o violáceas. Fruto legumbre o lomento. Hierbas postradas, rastreras o trepadoras volubles.

41. Hojas unifolioladas. Folíolo lanceolado-sagitado, de hasta $11 \times 8 \mathrm{~cm}$. Pecíolo alado. Flores blanco verdosas con guías de néctar lilas, de 3-3,5 cm long. Legumbre acuminada, rostrada, de 8-16 cm long., algunas de 3-4 cm long. Hierbas perennes rastreras o trepadoras volubles. 
41'. Hojas trifolioladas (a veces 4-5 folioladas en Centrosema variifolium). Folíolos no lanceolado-sagitados, menores. Pecíolo no alado. Flores amarillas, blanco-violáceas o violáceas. Fruto legumbre o lomento.

42. Hojas digitado-trifolioladas. Folíolos obovados a orbiculares, excepcionalmente elípticos. Racimos cortos. Flores amarillas de 1-1,5 cm long. Legumbre inflada, de 2-4 cm long. Hierbas postradas, pubescentes a glabrescentes, con pelos tiesos largos y amarillentos, más abundantes en el tallo.

Crotalaria incana ${ }^{\circ}$

42'. Hojas pinnado-trifolioladas. Folíolos elípticos a ovados. Racimos cortos o largos. Flores de diversos colores, si amarillas menores a $1,1 \mathrm{~cm}$ long. Legumbre no inflada o lomento. Hierbas postradas o rastreras o trepadoras volubles, pubescentes o glabras, si pubescentes con pubescencia no amarillenta.

43. Folíolos ovado-rómbicos, los laterales asimétricos. Legumbre globosa o plana. Trepadoras volubles.

44. Flores amarillas, pequeñas (de hasta $11 \mathrm{~mm}$ long.). Legumbres cortas y globosas, ligeramente comprimidas, de hasta $2,5 \mathrm{~cm}$ long. con 1-2 semillas.

45. Legumbre elipsoide. Flores de 6-11 mm long. Folíolos con glándulas sólo en la cara abaxial.

Rhynchosia edulis ${ }^{\circ}$

45'. Legumbre acinaciforme. Flores de 3-6 mm long. Folíolos con glándulas en ambas caras.

Rhynchosia minima ${ }^{\circ}$

44'. Flores blanco-verdosas o violetas, mayores. Legumbres largas y delgadas, rectas o falcadas, mayores a 2,5 cm long. Semillas más de 2.

46. Plantas pubescentes con pelos largos sedosos. Folíolo central frecuentemente pandurado. Ápice de la inflorescencia comoso (con grupo de brácteas subuladas y de borde ciliado formando un penacho). Flores violeta-rojizas, de 14-25 mm long., quilla espiralada. Legumbre subcilíndrica de 4-8 cm long.

Macroptilium bracteatum ${ }^{\circ}$

46'. Plantas glabras o escasamente pubescentes. Folíolo central ovado o elíptico. Racimos no comosos. Flores blanco-liláceas, menores, quilla recta o espiralada. Legumbre comprimida.

47. Flores blanco-verdosas, quilla verdosa con el extremo morado, espiralada formando 1-varios giros. Pedicelo de aproximadamente $1 \mathrm{~cm}$, más largo que el cáliz. Racimos con 1(2) flores por nudo. Legumbre acinaciforme de 3-4 cm long.

Phaseolus lunatus var. sylvester

47 '. Flores blancas o violáceas, quilla recta. Pedicelo menor a $3 \mathrm{~mm}$ long. Racimos con 3 o más flores por nudo. Legumbre recta mucronada, de 2,5-3,5 cm long.

Neonotonia wightii *

43'. Folíolos elípticos, ovados o redondeados, simétricos. Fruto legumbre o lomento. Hierbas postradas o rastreras o trepadoras volubles.

48. Lomento con pelos uncinados, artejos sumamente adherentes. Flores lila-violáceas menores a 1,5 cm long., no resupinadas. Estandarte sin espolón. Hierbas rastreras, postradas o ligeramente erectas. 


\section{A. V. Carranza et al. - Fabaceae de Selva Pedemontana de Jujuy}

49. Lomento con istmo marginal. Estípulas soldadas totalmente o sólo en la porción basal por el lado opuesto al pecíolo. Hojas inferiores a menudo unifolioladas, hojas superiores trifolioladas; folíolos subcoriaceos, obovados a elípticos, generalmente emarginados, los unifoliolados orbiculares; epifilo glabrescente con pelos uncinulados, hipofilo pubescente con pelos uncinulados y hialinos adpresos. Flores de 5-7 mm long. Tallos del año cilíndricos, glabros o ligeramente pubescentes, no adherentes.

Desmodium incanum ${ }^{\circ}$

49'. Lomento con istmo submarginal. Estípulas libres. Hojas todas trifolioladas; folíolos membranáceos, el terminal ovado-romboidal, los laterales elípticos o subagudos, epifilo e hipofilo pubescentes, con pelos uncinulados y hialinos largos. Flores de 9-11 mm long. Tallos del año trisurcados, con pelos uncinados, adherentes.

Desmodium subsericeum ${ }^{\circ}$

48'. Legumbre comprimida recta con rostro largo. Flores vistosas, mayores a $2 \mathrm{~cm}$ long., resupinadas por torsión del pedicelo. Estandarte orbicular amplio, azul o violáceo con centro blanco, pubescente, con espolón en zona media o basal cubierto por el cáliz. Trepadoras volubles.

50. Hojas siempre trifolioladas. Racimos 1-3(-5) floros, de 1-3(-6) cm long.

Centrosema virginianum ${ }^{\circ}$

50'. Hojas con 3-5 folíolos, a veces 3 folíolos con 1 folíolo lateral bilobado. Racimos (1-)3-5 floros, de (2,5)6-12 cm long.

Centrosema variifolium ${ }^{\circ}$

39'. Hierbas o subarbustos erectos, nunca trepadoras. Flores papilionadas o no.

51. Hojas pinnado-trifolioladas. Flores papilionadas, blancas o lilas, de 4-5 mm long.

52. Folíolos dentados, de 1,5-3 cm long. Utrículo ovoide, negruzco, de 2-3 mm long. Flores blancas. Racimos terminales de hasta $30 \mathrm{~cm}$ long. Hierbas anuales o bi-trienales, de hasta 2 $\mathrm{m}$ alt., aromáticas (presencia de cumarina), glabras.

Melilotus albus, trébol blanco de olor * 。

$52^{`}$. Folíolos enteros, de 3-11 cm long. Lomento con 3-6 artejos redondeados u ovados. Flores blanco-rosadas o violáceas. Panoja terminal amplia de hasta $50 \mathrm{~cm}$, hojosa. Hierbas perennes o subarbustos de hasta $2 \mathrm{~m}$ alt., no aromáticos, con pelos uncinulados, adherentes.

Desmodium tortuosum ${ }^{\circ}$

51'. Hojas pinnadas. Flores papilionadas o no, mayores a $5 \mathrm{~mm}$.

53. Flores amarillas, no papilionadas, con 10 estambres dispuestos en 3 grupos ( 3 estaminodios superiores, 4 estambres medianos y 3 estambres abaxiales largos o 2 largos y el central estéril). Legumbre cilíndrica o tetragonal delgada, generalmente arqueada. Folíolos ovallanceolados u obovados mayores a $1,5 \mathrm{~cm}$ lat.

54. Hojas 4-6 yugadas; folíolos oval-lanceolados agudos o acuminados, concolores, glabros; glándula gruesa en la base del pecíolo. Legumbre generalmente erguida y arqueada, cilíndrica, con márgenes pajizos y centro marrón, de 8-14 x 0,6-0,9(-1) $\mathrm{cm}$. Racimos axilares muy cortos o sub-umbelados, con (1-)2-5(-8) flores. Estambre abaxial central estéril. Hierbas anuales de 0,3-1,5 m alt.

Senna occidentalis

54'. Hojas (2)3 yugadas; folíolos obovados obtusos, discolores, glabros o pubescentes en ambas caras o sólo en epifilo; glándula raqueal (1-2) en el punto de inserción de los folíolos (nunca en el tercer par). Legumbre tardíamente dehiscente, ascendente recta o arqueada hasta casi semicircular, comprimida tetragonalmente, marrón oscura con vetas 
claras irregulares, más larga y delgada, de 7-16(-18) x 0,25-0,6 x 0,2-0,3 cm. Racimos subsésiles axilares con 1-2 flores. Estambres abaxiales fértiles 3. Hierbas monocárpicas anuales o plurianuales, de 0,3-2 $\mathrm{m}$ alt.

Senna obtusifolia ${ }^{\circ}$

53'. Flores papilionadas blanquecinas o amarillas. Legumbre plana o cilíndrica. Folíolos elíptico-lanceolados u oval-elípticos menores a $1,5 \mathrm{~cm}$ lat.

55. Plantas inermes. Flores blancas, blanco-amarillentas o blanco-rosadas, de 8-12 mm long. Legumbre plana y recta, rostro curvo. Racimos axilares 2-4 floros. Hojas con 6-14 pares de folíolos opuestos, de 5-11(-18) mm lat. Hierbas, subarbustos o arbustos gráciles, casi glabros a vilosos.

Coursetia hassleri ${ }^{\circ}$

55'. Plantas con aguijones en raquis foliar, raros en tallos. Flores amarillas de 2-2,5 cm long. Legumbre cilíndrica casi recta, con suturas marcadas, rostro recto. Racimos axilares 1-8 floros. Hojas con 25-35(-50) pares de folíolos subopuestos, de 3-6 mm lat. Subarbustos erectos de 1,8-2,5 m alt., glabros a subglabros.

Sesbania exasperata ${ }^{\circ}$

\section{Conclusiones}

El estudio sistemático del área permitió avanzar en el conocimiento de la diversidad de la zona, específicamente en este trabajo en relación a la familia Fabaceae. El registro de Centrosema variifolium, Sesbania exasperata, Vachellia albicorticata y Senna obtusifolia en Jujuy constituyen un hallazgo importante para la flora de la provincia.

Respecto a Erythrina mulungu, se deben continuar estudios para definir su origen en Jujuy. Así también, en relación a los otros taxa sería importante avanzar en las investigaciones, como por ejemplo respecto a Prosopis alba var. panta, Macroptilium lathyroides y Anadenanthera colubrina var. colubrina, profundizando la revisión de los Herbarios y las recorridas de colección. En este sentido, es importante continuar los estudios a campo y aumentar las colecciones de los herbarios, fundamentalmente del Herbario JUA que contribuye a la conservación de los registros a nivel provincial y regional.

El $53 \%$ de las especies nativas registradas en la zona y la única especie endémica se encuentran en el PN Calilegua, lo que indica que, si bien el parque ejerce una importante acción de conservación, sería importante ampliar la superficie ya sea con la misma o con otra categoría de conservación, o asignar nuevas zonas para áreas protegidas. Más aún, esto debe considerarse teniendo en cuenta los procesos de desmonte y cambio de uso de la tierra y cambio climático que están ejerciendo una presión dramática sobre los recursos naturales. Por otra parte, debe tenerse en cuenta que en este estudio el esfuerzo de colección se centró en el PN Calilegua, lo que podría estar presentando un sesgo en cuanto a la proporción de Fabaceae del área de estudio presentes en el parque. Esto queda evidenciado en la proporción de especies que son aportadas desde otras publicaciones de la familia al total consignado en este trabajo para el área.

La presencia de Melilotus albus y sobre todo de Leucaena leucocephala ssp. glabrata en el PN Calilegua es preocupante, ya que ambas especies son invasoras.

Aproximadamente un quinto de las especies encontradas se distribuyen sólo en Salta y Jujuy o Salta, Jujuy y Tucumán, lo que pone de manifiesto la importancia de la conservación de áreas en esta región de alta biodiversidad. Por otra parte, también son necesarias la ampliación de la evaluación y actualización de las listas rojas y la implementación de acciones de protección y restauración en consonancia con estas evaluaciones.

\section{Contribución DE LOS AUtORES}

AVC diseñó la investigación, revisó los ejemplares determinados y analizó los datos, LHA realizó la consulta de herbario, bases de 


\section{A. V. Carranza et al. - Fabaceae de Selva Pedemontana de Jujuy}

datos y elaboración de mapa, AEZ determinó los ejemplares coleccionados y revisó determinaciones previas del Herbario JUA. Todos los autores participaron en la colección de material de campo. AVC y LHA realizaron la escritura del manuscrito.

\section{Agradecimientos}

Los autores agradecen a la SECTER - UNJu, por el financiamiento de los siguientes proyectos que permitieron llevar adelante este trabajo: "Relevamiento de comunidades vegetales del PN Calilegua, Jujuy, Argentina". Primera y segunda etapa (Cód. A/0039 y A/0088), "Relevamiento de comunidades vegetales del Sector Este del PN Calilegua (Jujuy, Argentina) y estudios de los disturbios provocados por la actividad petrolera de dichas comunidades" (Cód.: A/0147). Al Dr. Osvaldo Ahumada y la Lic. Alicia Rotman del Herbario JUA y la Cátedra de Botánica General de la Facultad de Ciencias Agrarias de la UNJu, por apoyar la realización de la investigación. A las/ os revisoras/es anónimos del Boletín de la SAB por su aporte para mejorar el resultado final del manuscrito. A los guardaparques del PN Calilegua y a la Delegación Regional Noroeste de APN por la autorización y colaboración para los censos y colecciones en el Parque. A Isabel Zingariello, Nadia Paco, Hector Sato, Gabriel Rodríguez, Emanuel Fuentes, Pablo Echenique, Elsa Aguanta, Rodrigo Argañaraz, Belén Toscano, José Mendoza, Juan Visich, Guillermo Nieva, Joel Guevara Soto, Emilce Benitez y Juan Vilte por su ayuda en los viajes de colección y en las tareas de herborización $\mathrm{y}$ procesamiento de las colectas.

\section{Bibliografía}

ALTSCHUL, S. von R. 1964. A taxonomic study of the genus Anadenanthera. Contributions from the Gray Herbarium of Harvard University. 193: 3-65.

ANTON A. M. \& F. O. ZULOAGA (dirs.). 2019. Flora Argentina. Disponible en http://buscador. floraargentina.edu.ar/ [Acceso: 15 Noviembre 2019].

APN. 2009. Mapa de cobertura de suelo del Parque Nacional Calilegua. Sistema de Información de Biodiversidad, Administración de Parques Nacionales.
BLACKBURN, T. M, P. PYSEK, S. BACHER, J T. CARLTON, R. P. DUNCAN, V. JAROSIK, J. R. U. WILSON \& D. M. RICHARDSON. 2011. A proposed unified framework for biological invasions. Trends in Ecology and Evolution, 26: 333-339. https:/doi. org/10.1016/j.tree.2011.03.023

BOELCKE, O. 1946. Estudio morfológico de las semillas de Leguminosas Mimosoideas y Caesalpinioideas de interés agronómico en la Argentina. Darwiniana 7: 240-322.

BROWN, A. D. \& E. D. RAMADORI. 1988. Patrón de distribución, diversidad y características ecológicas de especies arbóreas de las Selvas y Bosques Montanos del Noroeste de Argentina. Actas del VI Congreso Forestal Argentino. Santiago del Estero: 177-180.

BURKART, A. 1937. Las especies argentinas del género Centrosema (Leguminosae). Darwiniana 3: 7-26.

BURKART, A. 1952. Las leguminosas argentinas silvestres $y$ cultivadas. $2^{\circ}$ ed. Acme, Buenos Aires.

BURKART, A. 1976. A monograph of the genus Prosopis (Leguminosae subfam. Mimosoideae). Journal of the Arnold Arboretum 57: 450-525.

CABRERA, A. L. 1976. Territorios fitogeográfico de la República Argentina. En: PARODI L. R. (ed.), Enciclopedia Argentina de Agricultura y Jardinería, 2: 2-85. Acme, Buenos Aires.

CASTRO, H. 1995. Una aproximación al estudio de las formas de ocupación y uso productivo del sector pedemontano de las Yungas. En: BROWN, A. D. \& H. R. GRAU (eds.), Investigación, Conservación y Desarrollo de Selvas Subtropicales de Montaña, Proyecto de Desarrollo Forestal, pp 215-222. LIEY, San Miguel de Tucumán.

CIALDELlA, A. M. 1984. El género Acacia (Leguminosae) en la Argentina. Darwiniana 25: 59-111.

CIALDELLA, A. M. 1997. Fabaceae, parte 4. Subfam II Mimosoideae, parte 2: Acacia. En: A. T. HUNZIKER (ed.), Fl. Fanerog. Argent. 35: 3-21.

DOCUMENTAE FLORAE AUSTRALIS 2020. Instituto Multidisciplinario de Biología Vegetal (IMBIV), Instituto Darwinion (IBODA), Instituto de Botánica del Nordeste (IBONE), INTA Castelar [Online]. Disponible en http://www.darwin.edu.ar/iris. [Acceso: 20 Enero 2020].

DREWES, S. I. 1995. Revisión de las especies argentinas del género Macroptilium (Leguminosae-Phaseolineae). Tesis doctoral. Universidad de Buenos Aires. Argentina.

DREWES, S. I. 1997. El género Macroptilium (Fabaceae) en la flora argentina. Bol. Soc. Argent. Bot. 32: 195-216. 
FORTUNATO, R. H. 1984. Dos leguminosas nuevas para la Flora Argentina. Darwiniana 25: 367-369.

FORTUNATO, R. H. 1986. Revisión del género Bauhinia (Cercideae, Caesalpinioidea, Fabaceae) para la Argentina. Darwiniana 27: 527-557.

FORTUNATO, R. H. \& E. A. ULIBARRI. 1997. Fabaceae. Cercideae. Detarieae. En: HUNZIKER, A. T. (ed.), $F l$. Fanerog. Argent. 33: 1-13.

GASPARRI, N. I. \& H. R. GRAU. 2009. Deforestation and fragmentation of Chaco dry forest in NW Argentina (1972-2007). Forest Ecology and Management 258: 913-921. https://doi.org/10.1016/j. foreco.2009.02.024

HOC, P. S. 1990. Las especies argentinas de Inga (Leguminosae, Mimosoideae). Darwiniana 30: 237 258.

HOC, P. S. 2005. Fabaceae. Mimosoideae. Ingeae. En: ANTON, A. M. \& F. O. ZULOAGA (eds.), $F l$. Fanerog. Argent. 93: 1-26.

HOC, P. S., R. A. PALACIOS \& M. P. MOM. 2006. Novedades en especies de Vigna e Inga (Leguminosae) para la Argentina. Bol. Soc. Argent. Bot. 41: 331-332.

LOZANO, E. C. \& M. A. ZAPATER. 2010. El género Erythrina (Leguminosae) en Argentina. Darwiniana 48: 179-200.

LPWG. THE LEGUME PHYLOGENY WORKING GROUP. 2017. A new subfamily classification of the Leguminosae based on a taxonomically comprehensive phylogeny. Taxon 66: 44-77. https://doi.org/10.12705/661.3

MARTÍNEZ, O. G., M. E. BARRANDEGUY, M. V. GARCÍA, D. A. CACHARANI \& D. E. PRADO. 2013. Presencia de Anadenanthera colubrina var. colubrina (Fabaceae, Mimosiodeae) en Argentina. Darwiniana nueva serie 1: 279-288. https://doi.org/10.14522/darwiniana.2013.12.536

MORELLO, J., S. D. MATEUCCI, A. F. RODRIGUEZ \& M. S. SILVA. 2012. Ecorregiones y complejos ecosistémicos argentinos. Orientación Gráfica Editora, Buenos Aires.

OYARZABAL, M., J. CLAVIJO, L. OAKLEY, F. BIGANZOLI, P. TOGNETTI, I. BARBERIS, H. M. MATURO, R ARAGON, P. I. CAMPANELLO, D. PRADO, M. OESTERHELD \& R. C. LEON. 2018. Unidades de Vegetación de Argentina. Ecología Austral 28: 40-63. https://doi.org/10.25260/EA.18.28.1.0.399

PALACIOS, R. A. \& M. M. BRIZUELA. 2005. Fabaceae, parte 13. Subfam II Mimosoideae, parte 4.Tribu VI. Mimoseae, parte B. Prosopis. En: ANTON, A. M. \& F. O. ZULOAGA (eds.), $F l$ Fanerog. Argent. 92: 3-24.

PALACIOS, R. A., P. S. HOC. \& S. I. DREWES. 2001. Fabaceae. Phaseoleae. Phaseolinae: Dolichopsis, Macroptilium, Phaseolus, Vigna. En: HUNZIKER, A. T. (ed.), Fl. Fanerog. Argent. 75: 1-18.

SARTORI, A. L. B., G. P. LEWIS, V. F. MANSANO \& A. M. G. AZEVEDO-TOZZI. 2015. A revision of the genus Myroxylon (Leguminosae: Papilionoideae). Kew Bull. 70: 1-12. https://doi.org/10.1007/s12225-015-9604-7

TROPICOS. 2020. Botanical Garden. Tropicos Database [Online]. Disponible en https://www.tropicos.org/ home [Acceso: 20 Enero 2020].

UICN. 2020. Rest List. Index [Online]. Disponible en https://www.iucnredlist.org/assessment/red-listindex [Acceso: 6 Febrero 2020].

ULIBARRI, E. A. 1996. Sinopsis de Caesalpinia y Hoffmannseggia (Leguminosae-Caesalpinioiedeae) de Sudamérica. Darwiniana 34: 229-348.

ULIBARRI, E. A. 2004. Leucaena leucocephala (Leguminosae-Mimosoideae) adventicia en Argentina. Hickenia 3: 221-224.

VOLANTE, J. N., A. R. BIANCHI, H. P. PAOLI, Y. E. NOE, H. J. ELENA \& C. M. CABRAL. 2005. Análisis de la dinámica del uso del suelo agrícola del Noroeste Argentino mediante teledetección $y$ sistemas de información geográfica. Periodo 2000-2005. Instituto Nacional de Tecnología Agropecuaria.

ZALlOCCHI, E. M., A. B. POMILIO \& R. A. PALACIOS. 1995. Estudio quimiotaxonómico de la Subtribu Phaseolinae (Phaseoleae-PapilionoideaeLeguminosae) III: Flavonoides de las especies argentinas de los géneros Phaseolus y Dolichopsis. Darwiniana 33: 135-148.

ZAPATER, M. A, P. S. HOC, E. C. LOZANO \& S. S. SÜHRING. 2014. Delimitación de las especies argentinas del género Inga (Mimosoideae) mediante técnicas numéricas. Darwiniana nueva serie 2: 248 259. https://doi.org/10.14522/darwiniana.2014.22.614

ZULOAGA, F. O. \& M. J. BELGRANO (eds.). 2020. Catálogo de las Plantas Vasculares del Cono Sur (Argentina, Sur de Brasil, Chile, Paraguay y Uruguay) [Online]. Disponible en http://www. darwin.edu.ar/Proyectos/FloraArgentina/fa.htm [Acceso: 20 Enero 2020]. 


\section{A. V. Carranza et al. - Fabaceae de Selva Pedemontana de Jujuy}

\section{ANEXO 1}

\section{Material examinado}

Amburana cearensis (Allemão) A.C. Sm.: Dpto. Ledesma: Parque Nacional Calilegua, seccional Aguas Negras, zona mirador, 25-III-2011, Carranza et al. 2366 (JUA).

Anadenanthera colubrina (Vell.) Brenan var. cebil (Griseb.) Altschul: Dpto. Ledesma: Parque Nacional Calilegua, Arroyo Aguas Negras Sur, 2353,273' S 645ㄷㄴ,267' W 843 msnm, 04-XII-2007, Carranza et al. 1328 (JUA). Dpto. San Pedro: Santa Clara, 2801-1980, Breglia 237 (JUA). Dpto. Santa Bárbara: Vinalito, Loc. Lote 537, 20-XI-2010, Carranza 2150 (JUA).

Cascaronia astragalina Griseb.: Dpto. Ledesma: Parque Nacional Calilegua, Sector Este. Subsector Caimancito, 2339'33,6” S 64³4'42,2” W 433 msnm, 09-III-2014, Carranza et al. 3547 (JUA); Parque Nacional Calilegua, Ruta 83, 2345'30,1" S 6450'58.4” W 615 msnm, 12-X-2013, Carranza et al. 3284 (JUA).

Centrosema sagittatum (Humb. \& Bonpl. ex Willd.) Brandegee ex L. Riley: Dpto. Ledesma: Parque Nacional Calilegua, Arroyo Aguas Sur, 2352'14,9' S 6455'47,7’' W 902 msnm, 03-III-2009, Carranza et al. 1569 (JUA). Dpto. Santa Bárbara: Palma Sola, 08-III-1983, Rotman et al. 689 (JUA).

Centrosema variifolium Burkart: Dpto. Ledesma: Parque Nacional Calilegua, Arroyo Aguas Negras Sur, 2354'20,7” S 6456’30,6” W 822 msnm, 03-III2009, Carranza et al. 1636 (JUA).

Centrosema virginianum (L.) Benth.: Dpto. Ledesma: Parque Nacional Calilegua, Arroyo Aguas Negras Sur, 2353'53,2” S 6456’24,7” W 816 msnm, 06III-2009, Carranza et al. 1650 (JUA). Dpto. Santa Bárbara: Vinalito, 30-III-1983, Ahumada 4673 (JUA).

Chloroleucon tenuiflorum (Benth.) Barneby \& J.W. Grimes: Dpto. Ledesma: entrada al Parque Nacional Calilegua, Ruta 83, 2347'17,69” S 6447'42,23” W 515 msnm, 10-X-2014, Carranza et al. 3703 (JUA). Dpto. San Pedro: San Pedro de Jujuy, 10-XI-1993, Lázzaro 01 (JUA).

Coursetia brachyrhachis Harms: Dpto. Ledesma: Parque Nacional Calilegua, Arroyo Aguas Negras Sur, 235' 59,40" S 6456'16,62” W 820 msnm, 06-XII2007, Carranza et al. 1351 (JUA). Dpto. San Pedro: Las Lajitas, 19-IX-1981, Vignale 339 (JUA).
Coursetia hassleri Chodat: Dpto. Ledesma: Parque Nacional Calilegua, sector Ruta 83, 2347'15,9' S 644' 58,4” W 525 msnm, 19-XII-2016, Carranza et al. 4103 (JUA); Parque Nacional Calilegua, Sector Este Subsector Caimancito, 233'28,4” S 643'이, ' W 580 msnm, 23-III-2010, Carranza et al. 1905 (JUA).

Crotalaria incana L.: Dpto. Ledesma: Parque Nacional Calilegua, Playa de Arroyo Aguas Negras Sur, 235, 594' S 6456,412' W 836 msnm, 21-X2005, Carranza et al. 976 (JUA). Dpto. San Pedro: Chaguaral, 18-IV-1983, Ahumada \& Castellón 4833 (JUA). Dpto. Santa Bárbara: Vinalito, 30-III1983, Ahumada 4654 (JUA).

Crotalaria micans Link: Dpto. San Pedro: Los Toldos, 11/VI/1987, Ahumada \& Castellón 5682 (JUA).

Desmanthus acuminatus Benth.: Dpto. San Pedro: Estación San Lucas, 500 - 600 msnm., 21-IV-1981, Ahumada 4218 (JUA, SI).

Desmanthus virgatus (L.) Willd.: Dpto. Ledesma: Parque Nacional Calilegua, Caimancito, 2337'18,59” S 643'ㄹ, $35^{\circ}$ "W 522 msnm, 28XII-2013, Carranza et al. 3467 (JUA).

Desmodium bridgesii (Schindl.) Burkart: Dpto. Ledesma: Parque Nacional Calilegua, Arroyo Sauzalito, $23^{\circ} 37^{\prime} 25,2^{\prime}$ 'S 64³5'21,7'” W 620 msnm, 17-X-2006, Carranza et al. 1024 (JUA).

Desmodium incanum DC.: Dpto. Ledesma: Parque Nacional Calilegua, Sector Este, Subsector Caimancito, $23^{\circ} 38^{\prime} 31,4$ " S 64³5'52,8” W 550 msnm, 22-III-2010, Carranza et al. 1841 (JUA).

Desmodium subsericeum Malme: Dpto. Ledesma: Parque Nacional Calilegua, Subsector Caimancito, 2337’31,2” S 64³6'9,5” W 18-X-2006, Carranza et al. 1076 (JUA).

Desmodium tortuosum (Sw.) DC.: Dpto. Ledesma: Parque Nacional Calilegua, Sector Este, Subsector Caimancito, $23^{\circ} 38^{\prime} 46,4^{\prime \prime}$ S 64³5'30,9” W 501 msnm, 24-III-2011, Carranza et al. 2338 (JUA).

Enterolobium contortisiliquum (Vell.) Morong: Dpto. Ledesma: Parque Nacional Calilegua, Arroyo Aguas Negras Sur, 2353'54,4”'S 6456'21,1" W 805 msnm, 14-XI-2008, Carranza et al. 1478 (JUA).

Erythrina crista-galli L. var. crista-galli: San Pedro: El Quemado, 246'24,90” S 6449'38,52” W 445 msnm., 06-IX-2018, Carranza et al. 4489 b (JUA). 
Erythrina mulungu Mart.: Dpto. Ledesma: Finca Yuchán del Ingenio Ledesma, 2356'539” S 6452’705” W 200 msnm, 21-X-2005, Carranza et al. 990 (JUA). Dpto. San Pedro: Ingenio La Esperanza, 12-XI1981, Rotman 621 (JUA).

Geoffroea decorticans (Gillies ex Hook. \& Arn.) Burkart var. decorticans: Dpto. Ledesma: Camino a Parque Nacional Calilegua, Ruta 83, 2347'17,69” S 6447'42,23' W 515 msnm, 10-X-2014, Carranza et al. 3716 (JUA). Dpto. San Pedro: márgenes del río San Pedro, 650 msnm, 16-IX-1971, Legname \& Cuezzo 8590 (JUA).

Gleditsia amorphoides (Griseb.) Taub. var. amorphoides: Dpto. Ledesma: Parque Nacional Calilegua, 23⒌'16,40" S 64'57'27,45" W 855 msnm, 15X-2004, Carranza et al. 928 (JUA). Dpto. Santa Bárbara: alrededores de Finca La Campana, 24¹6' S 6414' W 800 msnm, 12-XII-1998, Ahumada et al. 8889 (JUA).

Inga marginata Willd.: Dpto. Ledesma: Parque Nacional Calilegua, Sector Caimancito, 2337'31,2" S 64³6’9,5” W 18-X-2006, Carranza et al. 1080 (JUA); Parque Nacional Calilegua, orillas Arroyo Aguas Negras, 234' S 6450' W 720 msnm, 02-XI1998, Ahumada \& Agüero 8523 (JUA).

Inga saltensis Burkart: Dpto. Ledesma: camino al Arroyo Cafetal, 233' S 644' W 860 msnm, 04-XI-1998, Ahumada \& Agüero 8597 (JUA).

Leucaena leucocephala (Lam.) de Wit ssp. glabrata (Rose) Zárate: Dpto. Ledesma: Parque Nacional Calilegua, Yacimiento Caimancito, Este Caimancito, Pozo 40, 23 38' 31,4”' S 64 35' 52, $8^{\circ}$ ' W 550 msnm, 18-III-2020, Carranza et al. 4680 (JUA). Dpto. San Pedro: El Cuarteadero, 24²1'31,6" S 6457'49,1" W 836 msnm, 23-X-2017, Carranza 4678 (JUA). Dpto. Santa Bárbara: El Piquete, 05-VII-1977, Breglia 148 (JUA).

Libidibia paraguariensis (D. Parodi) G.P. Lewis: Dpto. San Pedro: Autódromo "Tierra Brava", 06-XI-2003, Álvarez 252 (JUA). Dpto. Santa Bárbara: Vinalito, 28-V-2011, Carranza 2463 (JUA).

Lonchocarpus lilloi (Hass1.) Burkart: Dpto. Ledesma: Parque Nacional Calilegua. Yacimiento Caimancito, $23^{\circ} 37^{\prime} 36,21^{\prime}$ S $64^{\circ} 35^{\prime} 16,36$ " W 522 msnm, 27XII-2013, Carranza et al 3455 (JUA).

Macroptilium bracteatum (Nees \& C. Mart.) Maréchal \& Baudet: Dpto. Ledesma: Parque Nacional Calilegua, Arroyo Aguas Negras Sur, 2352'54,3' S 6456'7,5' W 861 msnm, 04-III-2009, Carranza et al. 1529 (JUA).
Melilotus albus Desr.: Dpto. Ledesma: Parque Nacional Calilegua, Sector Este, Caimancito, 23³7'45,2” S 643'ㅇ, '” W 510 msnm, 10-IX-2010, Carranza et al. 2077 (JUA).

Mimosa debilis Humb. \& Bonpl. ex Willd. var. debilis: Dpto. Ledesma: Parque Nacional Calilegua, Arroyo Aguas Negras Sur, 2354'20,7’' S 6456'30,6” W 822 msnm, 02-III-2009, Carranza et al. 1620 (JUA).

Mimosa polycarpa Kunth var. subandina Barneby: Dpto. Ledesma: Parque Nacional Calilegua, zona de Arroyo Aguas Negras Sur, 2354'20,7’'S 6456’30,6” W 822 msnm, 02-III-2009, Carranza et al. 1511 (JUA). Dpto. Santa Bárbara: alrededores de Finca La Campana,

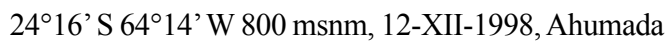
et al. 8969 (JUA).

Mimosa sensibilis Griseb. var. sensibilis: Dpto. Ledesma: camino al Parque Nacional Calilegua, 14-II-1996, Ahumada \& Toppano 7357 (JUA).

Myroxylon peruiferum L. f.: Dpto. Santa Bárbara: Ruta Provincial $\mathrm{N}^{\circ} 6$, de Santa Clara a El Fuerte, $24^{\circ} 18^{\prime}$ S 643' W 840 msnm, 25-IX-2019, Carranza et al. 4654 (JUA).

Neonotonia wightii (Graham ex Wight \& Arn.) Lackey: Dpto. Santa Bárbara: Santa Clara, 15-V-1983, Ahumada \& Castellón 4686 (JUA, SI).

Nissolia fruticosa Jacq. var. guatemalensis (Rose) Rudd: Dpto. Ledesma: Parque Nacional Calilegua, Sector Este Caimancito, 2337’23,10”'S 64³5'20,30”' W 15 II-2017, Carranza 4162 (JUA).

Parapiptadenia excelsa (Griseb.) Burkart: Dpto. Ledesma: Parque Nacional Calilegua, Sector Caimancito, 2338'12,4”' S 64³5'56,8” W 466 msnm, 04-II-2010, Carranza et al. 1775 (JUA).

Phaseolus lunatus L. var. silvester Baudet: Dpto. Ledesma: entrada al Parque Nacional Calilegua, Sector Ruta Provincial N 83, 2347'18,8” S 64²7’28,8”' W 519 msnm, 15-II-2017, Carranza et al. 3617 (JUA). Dpto. Santa Bárbara: Vinalito, 30-II-1983, Ahumada 4674 (JUA).

Poissonia hypoleuca (Speg.) Lillo: Dpto. Ledesma: Parque Nacional Calilegua, Sector Ruta 83, Arroyo Aguas Negras, 2345'38,5”'S 6450'58,8”'W 612 msnm, 12X-2013, Carranza et al. 3261 (JUA).

Prosopis alba Griseb. var. alba: Dpto. Ledesma: Loc. Palo Blanco, 2355'14,3” S 6450’30,74” W 499 msnm, 06-XI-2018, Carranza et al. 4499 (JUA). Dpto. San Pedro: Ruta Nacional $\mathrm{N}^{\circ} 34$, entrada a la ciudad de San Pedro, 24¹4'30,7’' S 6453'27'” W 630 msnm, 23-X-2017, Carranza 4679 (JUA). Dpto. Santa Bárbara: Vinalito, 21-XI-2010, Carranza 2160 (JUA). 


\section{A. V. Carranza et al. - Fabaceae de Selva Pedemontana de Jujuy}

Rhynchosia edulis Griseb.: Dpto. Ledesma: Parque Nacional Calilegua, Arroyo Aguas Sur, 235' $27,2^{\prime \prime}$ S 645' $18,7^{\prime \prime}$ W 840 msnm, 10-XI-2008, Carranza \& Sato 1442 (JUA). Dpto. Santa Bárbara: camino a Infiernillo, $24^{\circ} 17^{\prime} \mathrm{S} 64^{\circ} 14^{\prime} \mathrm{W} 780 \mathrm{msnm}, 14-\mathrm{XII}-$ 1998, Rotman 1276 (JUA).

Rhynchosia minima (L.) DC: Dpto. Ledesma: Parque Nacional Calilegua, Caimancito, Sector Este, 233'05,3" S 6435'57,6” W 597 msnm, 07-III2014, Carranza et al. 3503 (JUA). Dpto. San Pedro: Chaguaral, 22-II-1983, Ahumada \& Castellón 4810 (JUA).

Senegalia parviceps (Speg.) Seigler \& Ebinger: Dpto. Ledesma: Parque Nacional Calilegua, Arroyo Aguas Negras Sur, 2353,99' S 6456,277' W 850 msnm, 06-XII-2007, Carranza 1356 (JUA). Dpto. San Pedro: Las Lajitas, 05-III-1980, Vignale \& Breglia 309 (JUA).

Senegalia tucumanensis (Griseb.) Seigler \& Ebinger: Dpto. Ledesma: Parque Nacional Calilegua, Arroyo

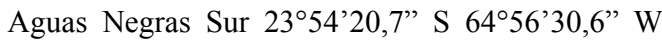
822 msnm, 02-III-2009, Carranza et al. 1493 (JUA).

Senna morongii (Britton) H.S. Irwin \& Barneby: Dpto. Santa Bárbara: Vinalito. Lote 1, 2339'8,10" S 64²0'13,39” W 327 msnm, 25-V-2019, Carranza et al. 4587 (JUA). Dpto. San Pedro: Estación San Lucas, 500 - 600 msnm, 21-IV-1981, Ahumada 4211 (JUA).

Senna obtusifolia (L.) H.S. Irwin \& Barneby: Dpto. Ledesma: Parque Nacional Calilegua, Sector Este, Subsector Caimancito, $23^{\circ} 38^{\prime} 25,4^{\prime \prime}$ S 643'․' W 570 msnm, 22-III-2010, Carranza et al. 1853 (JUA); Parque Nacional Calilegua, área afectada al Yacimiento Petrolífero Caimancito de Pluspetrol

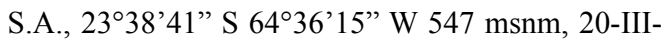
2008, Tolaba 4945 (JUA). Dpto. Santa Bárbara: Palma Sola, 12-V-1983, Ahumada \& Castellón 4943 (JUA).

Senna occidentalis (L.) Link: Dpto. Ledesma: camino al Parque Nacional Calilegua, 29-II-1996, Ahumada \& Toppano 7354 (JUA). Dpto. San Pedro: Acheral, 21IV-1988, Ahumada \& Castellón 5759 (JUA).

Senna pendula (Willd.) H.S. Irwin \& Barneby var. eriocarpa (Griseb.) H.S. Irwin \& Barneby: Dpto. Ledesma: Parque Nacional Calilegua, Sector Este,

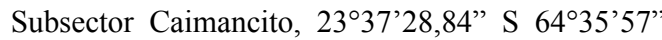
W 569 msnm, 28-XII-2011, Carranza et al. 2672 (JUA). Dpto. Santa Bárbara: Vinalito, 30-III-1983, Ahumada 4664 (JUA).

Senna spectabilis (DC.) H. S. Irwin \& Barneby var. spectabilis: Dpto. Ledesma: Parque Nacional Calilegua, Sector Sur, zona Rio Colorado, Arroyo Aguas Negras Sur, 2353'49,1' S 6456'26” W 830 msnm, 03-III-2009, Carranza et al. 1526 (JUA). Dpto. Santa Bárbara: Palma Sola, 08-III-1983, Rotman et al. 687 (JUA).

Sesbania exasperata Kunth: Dpto. Ledesma: Parque Nacional Calilegua, Sector Este. Subsector

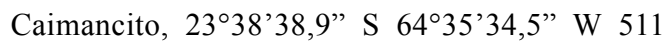
msnm, 22-III-2013, Carranza et al. 3172 (JUA).

Tipuana tipu (Benth.) Kuntze: Dpto. Ledesma: Parque Nacional Calilegua, Arroyo Aguas Negras Sur, 2353.99" S 645 57.277" W 850 msnm, 07-XII-2007, Carranza et al. 1361 (JUA).

Vachellia albicorticata (Burkart) Seigler \& Ebinger: Dpto. Ledesma: Parque Nacional Calilegua, Caimancito, $23^{\circ} 38,242^{\prime}$ S 643ㅜㅇ, 873' W 587 msnm, 05-XI-2009, Carranza 1734 (JUA). Dpto. Santa Bárbara: Vinalito, 2340'13,13" S 64²1'21,64” W 19-VII-2018, Carranza et al. 4638 (JUA).

Vachellia aroma (Gillies ex Hook. \& Arn.) Seigler \& Ebinger: Dpto. Ledesma: Parque Nacional Calilegua, Sector Este, Subsector Caimancito, 2338'31,4” S

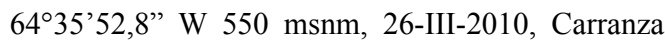
1957 (JUA). Dpto. San Pedro: Las Lajitas, 28-II1975, García 528 (JUA). Dpto. Santa Bárbara: camino a Infiernillo, $24^{\circ} 17^{\prime} \mathrm{S} 64^{\circ} 14^{\prime} \mathrm{W} 780 \mathrm{msnm}$, 14-XII-1998, Ahumada et al. 9008 (JUA).

Vachellia macracantha (Humb. \& Bonpl. ex Willd.) Seigler \& Ebinger: Dpto. Ledesma: Parque Nacional Calilegua, sendero a La Lagunita, $23^{\circ} 45^{\prime} 11,40^{\prime \prime} \mathrm{S}$ 6450'55,20”' W 650 msnm, 01-XI-1998, Ahumada \& Agüero 8453 (JUA). Dpto. Santa Bárbara: Palma Sola, 08-III-1983, Ahumada et al. 4509 (JUA).

Zapoteca formosa (Kunth) H. M. Hern.: Dpto. Ledesma: Parque Nacional Calilegua, Sector Este Caimancito, $23^{\circ} 38^{\prime} 45,2^{\prime \prime} \mathrm{S} 64^{\circ} 35^{\prime} 55,1^{\prime \prime} \mathrm{W} 508 \mathrm{msnm}, 26-\mathrm{XII}-$ 2013, Carranza et al. 3398 (JUA). Dpto. Santa Bárbara: camino a Infiernillo, $24^{\circ} 17^{\prime}$ S $64^{\circ} 14^{\prime}$ W 780 msnm, 14-XII-1998, Ahumada et al. 9011 (JUA). 


\title{
ANEXO 2
}

\author{
Ejemplares de especies de la zona de estudio publicadas por otros autores \\ (se respeta el formato de cita original) \\ *: especie introducida, ${ }^{\circ}$ : especie presente en el PN Calilegua
}

Anadenanthera colubrina (Vell.) Brenan var. colubrina: Novara 11073 (MCNS 11393); Del Castillo 460 (MCNS 11363); Legname s/n (MCNS 11406; Vignale 340 (MCNS 11384): Martinez et al. (2013).

Arachis duranensis Krapov. \& W.C. Greg.: Schinini \& al. 19539 (CTES); Krapovickas \& al. 38905 (CTES): Krapovickas \& Gregory (2007).

Bauhinia forficata Link ssp. pruinosa (Vogel) Fortunato \& Wunderlin *: Spegazzini 13822 (BAB): Fortunato (1986), Fortunato \& Ulibarri (1997).

Bauhinia mollis (Bong.) D. Dietr. var. notophila (Griseb.) Fortunato: Cabrera et al. 31492 (SI): Fortunato (1986), Gamerro \& Fortunato (2001), Zapater \& Lozano (2012). Cabrera et al. 31492 (MBM): Fortunato (1986). Fabris 3143 (BAB, LP); Rial Alberti 415 (BAB): Fortunato (1986), Fortunato \& Ulibarri (1997). Novara et al. 12169 (MCNS); Zapater \& Lozano 2840a, 2840b (MCNS): Zapater \& Lozano (2012). Fabris et al. 3143 (SI): Fortunato (1986), Fortunato \& Ulibarri (1997), Zapater \& Lozano (2012).

Caesalpinia pulcherrima (L.) Sw.: Burkart 11415 (SI): Boelcke (1946).

Chloroleucon chacöense (Burkart) Barneby \& J.W. Grimes: Spegazzini s/n (LP 24390): Hoc (2005). Citada como Pithecellobium chacoense Burkart: Spegazzini s/n (LP 24390): Hoc (1981, 1982).

Chloroleucon foliolosum (Benth.) G.P. Lewis: Cabrera 21110 (SI): Hoc (2005). Citada como Pithecellobium grisebachianum Harms: Schuel s/n (BAB): Hoc (1981). L. Cabrera y H. A. Fabris 21110 (LP): Hoc (1981, 1982). Citada como Calliandra grisebachiana (Harms) Speg. var. carolae Speg.: Speggazzini s/n (LPS 11909): Gutierrez et al. (2002).

Cochliasanthus caracalla (L.) Trew: Citada como Vigna caracalla (L.) Verdcourt: O. Ahumada 4650 (UNJ-FCA): Di Stilio (1994). Observaciones: Este ejemplar no se encuentra en el Herbario JUA.

Condylostylis candida (Vell.) A. Delgado: Citada como Vigna candida (Vell.) Maréchal, Mascherpa \& Stainier: Ahumada 4623 (UNJ, FCA, SI): Hoc et al. (2006). Observaciones: Este ejemplar no se encuentra en el Herbario JUA.
Crotalaria chaco-serranensis $\mathrm{H}$. Bach \& Fortunato: R. Kiesling \& al. 5577 (BAB, SI); H. A. Fabris 4538 (BAB); A. L. Cabrera et al. 14481 (BAB): Bach \& Fortunato (2010).

Crotalaria maypurensis Kunth: Fabris 5295 (LP, SI): Gomez Sosa (2000a).

Crotalaria pumila Ortega: Cabrera et al. 30257 (SI): Gomez Sosa (2000a).

Desmanthus paspalaceus (Lindm.) Burkart: Citada como Desmanthus paspalaceus (Lindman) Burkart: Venturi 5364 (F, GH, MO, NY, UC, US); Venturi 5368 (CAS): Luckow (1993).

Desmodium affine Schltdl.: O. Ahumada 4671 (CTES): Vanni (2001).

Desmodium glabrum (Mill.) DC.: Cabrera et al. 32752 (CTES, SI); Burkart 20150 (SI): Vanni (2001).

Desmodium procumbens (Mill.) Hitchc. Citada como Desmodium neo-mexicanum A. Gray. Krapovickas et al. 39299 (CTES): Vanni (2001).

Erythrostemon argentinus (Burkart) E. Gagnon \& G. P. Lewis: Spegazzini 2159 (holotype LP, isotype SI): Gagnon et al. (2016). Citada como Caesalpinia argentina Burkart: Devoto y Alberti, Hb Min Agric Gand 2373 (SI); Cabrera 20837 (SI); Guaglianone et al. 2571 (SI): Ulibarri (1996).

Geoffroea decorticans (Gillies ex Hook. \& Arn.) Burkart var. subtropicalis (Lillo) Burkart: Venturi 5251 (SI); Spegazzini s/n (LPS 18493): Burkart (1949).

Inga edulis Mart.: Citada como Inga edulis C. Martius var. parviflora Benth.: Cabrera y Fabris 21114 (CTES, LP); Cabrera et al. 32277, 30966 (SI); Jörgensen-Hansen 35911 (BAB): Hoc (1990). Cabrera et al. 21114 (SI); Lillo 5124 (LIL): Hoc (1990, 2005).

Leptospron adenanthum (G. Mey.) A. Delgado *: Citada como Vigna adenantha (G. F. Meyer) Maréchal, Mascherpa et Stalnler: Palacios 1209 (BAFC): Zallocchi et al. (1993), Di Stilio (1994), Espert \& Burghard (2003).

Macroptilium lathyroides (L.) Urb.: Vervoorst et al. 4667 (SI) o Vervoost \& Baciagalupo 4667 (SI): Drewes (1995 y 1997), Palacios et al. (2001). Observación: este ejemplar presenta problemas, como se indica en cuerpo principal. 


\section{A. V. Carranza et al. - Fabaceae de Selva Pedemontana de Jujuy}

Macroptilium panduratum (C. Mart. ex Benth.) Maréchal \& Baudet: Citada como Macroptilium panduratum (Benth.) Maréchal \& Baudet: Fernandez 1624 (SI, BAFC): Drewes (1995 y 1996). Hoc 66 (BAFC): Zallocchi et al. (1992), Drewes (1995, 1996 y 1997). Burkart 20149 (SI); Cabrera 23340 (LP); Cabrera et al. s/n 34674 (SI): Drewes (1995). Fernandez 1624 (CTES): Drewes (1995).

Mimosa farinosa Griseb. ${ }^{\circ}$ : Venturi 5230 (SI, LIL): Burkart (1948).

Mimosa xanthocentra Mart. var. xanthocentra: Citada como Mimosa stenoptera Benth.: Schreiter 5369 (LIL); Spegazzini 15723 (BAB): Burkart (1948), Caccavari (1985).

Mimozyganthus carinatus (Griseb.) Burkart: Schuel 87 (Buen Mus); Spegazzini 10945 (LPS): Burkart (1939a). Observación: probablemente Buen Mus corresponde a BA.

Parasenegalia visco (Lorentz ex Griseb.) Seigler \& Ebinger: Citada como Acacia visco Lor. ex Grisebach: Venturi 5382 (BAB, SI): Cialdella (1984).

Parkinsonia aculeata L.: Cabrera \& H. Fabris 16169, 16234 (LP): Ulibarri (2008).

Parkinsonia praecox (Ruiz \& Pav. ex Hook.) Hawkins ○: Citada como Cercidium praecox (Ruiz et Pavón) Harms subsp. praecox: W. J. Eyerdam \& A. A. Beetle 22503 (UC, MO); A. L. Cabrera 16067 (F); S. Venturi 5343 (CAS, F, UC, US, MO, SI): Burkart \& Carter (1976).

Phaseolus lunatus L.: Ahumada 4674 (LIL, dupl. BAFC); Palacios 1210 (BAFC): Zallocchi et al. (1995).

Prosopis alba Griseb. var. panta Griseb.: Carmelich \& Rial Alberti Herb. Forestal 580 (SI): Burkart (1976). Observación: ver análisis en Resultados y Discusión.

Prosopis nigra (Griseb.) Hieron.: Citada como Prosopis nigra (Grisebach) Hieronymus: Killip 39632 (SI, US); Eyerdam \& Beetle 22534 (MO): Burkart (1976).

Pterogyne nitens Tul.: Cabrera et al.31422 (SI): Ulibarri (1997). Guaglianone et al. 1820 (SI); Taylor et al. 11445 (SI): Ulibarri (2008).

Rhynchosia naineckensis Fortunato: Burkart et al. 30470 (SI); Cabrera et al. 21553, 31941 (SI); Correa et al. 4573 (BAB); Fabris 8248 (SI); Jones s/n (BAB, 10435 SI); de la Sota 4393 (LP, SI); Zuloaga \& Deginani 142 (LP): Fortunato (1983). Observación: no se encontraron las ubicaciones en Jujuy de estos ejemplares. Cabrera et al. 28461 (SI): Fortunato (1983). Observación: en el Herbario SI figura como procedente de Misiones, Dpto. Apóstoles.
Senegalia etilis (Speg.) Seigler \& Ebinger: Citada como Acacia etilis Speg.: Fabris 5277 (LP, SI): Fortunato \& Cialdella (1997). Citada como Acacia etilis Spegazzini: Cabrera 24229, 31021 (SI); Fabris et al. 5277 (LP, SI): Cialdella (1984).

Senegalia praecox (Griseb.) Seigler \& Ebinger ${ }^{\circ}$ : Citada como Acacia praecox Grisebach: Cabrera 20808, 25741 (LP); Cabrera 32083 (SI); Fabris 4579 (LP); Venturi 5232 (SI): Cialdella (1984).

Sesbania bispinosa (Jacq.) W. Wight *: Citada como Sesbania bispinosa (Jacq.) Wight var. micrantha. Cabrera et al. 31366 (SI); Rossow et Fortunato 732 (BAB): Fortunato (1984), Gómez Sosa (2000b).

Stylosanthes scabra Vogel: Spegazzini (Min. Agr. 13943) (SI): Burkart (1939b), Vanni (2017). Observaciones: en SI figura un ejemplar compatible con este bajo “Spegazzini s/n SI 5874”. Spegazzini 2196: Burkart (1939b).

Vicia andicola Kunth: Ahumada 5345 (CTES): Vanni \& Kurtz (2005).

Vigna luteola (Jacq.) Benth *: Novara \& Subils 2745 (S, SI): Hoc et al. (2006).

\section{Bibliografía}

BACH, H. G. \& R. H. FORTUNATO. 2010. Crotalaria chaco-serranensis $\mathrm{H}$. Bach \& Fortunato nom. nov. (Leguminosae) del Chaco Argentino. Candollea 65: 291-300. https://doi.org/10.15553/c2010v652a8

BOELCKE, O. 1946. Estudio morfológico de las semillas de Leguminosas Mimosoideas y Caesalpinioideas de interés agronómico en la Argentina. Darwiniana 7: 240-322.

BURKART, A. 1939a. Descripción de Mimozyganthus, nuevo género de Leguminosas y sinopsis preliminar de los géneros Argentinos de Mimosóideas. Darwiniana 3: 445-469.

BURKART, A. 1939b. Estudios sistemáticos sobre las Leguminosas-Hedisareas de la República Argentina y regiones adyacentes. Darwiniana 3: 117-302.

BURKART, A. 1948. Las especies de Mimosa de la Flora Argentina. Darwiniana 8: 9-231.

BURKART, A. 1949. La posición sistemática del "chañar" y las especies del género Geoffroea (Leguminosae-Dalbergieae). Darwiniana 9: 9-23.

BURKART, A. 1976. A monograph of the genus Prosopis (Leguminosae subfam. Mimosoideae). Journal of the Arnold Arboretum 57: 450-525. 
BURKART, A. \& A. CARTER. 1976. Notas en el género Cercidium (Caesalpinoideae) en Sud América. Darwiniana 20: 305-311.

CACCAVARI, M. A. 1985. Granos de polen de leguminosas de la Argentina IV. Género Mimosa. Bol. Soc. Argent. Bot. 24: 151-167.

CIALDELlA, A. M. 1984. El género Acacia (Leguminosae) en la Argentina. Darwiniana 25: 59-111.

DI STILIO, V. S. 1994. Vigna (Leguminosae, Phaseoleae) en la Argentina: Estudio palinológico. Bol. Soc. Argent. Bot. 30: 3-11.

DREWES, S. I. 1995. Revisión de las especies argentinas del género Macroptilium (LeguminosaePhaseolineae). Tesis doctoral. Universidad de Buenos Aires. Argentina.

DREWES, S. I. 1996. Estudio palinológico de las especies argentinas de Macroptilium (Fabaceae). Darwiniana 34: 233-244.

DREWES, S. I. 1997. El género Macroptilium (Fabaceae) en la flora argentina. Bol. Soc. Argent. Bot. 32: 195216.

ESPERT, S. M. \& A. D. BURGHARDT. 2003. Electrophoretic analysis of seed proteins in Argentinean species of Phaseolinae (Fabaceae). Bol. Soc. Argent. Bot. 38: 311-317.

FORTUNATO, R. H. 1983. Sinopsis de las especies argentinas del género Rhynchosia. Parodiana 2: 25-58.

FORTUNATO, R. H. 1984. Dos leguminosas nuevas para la Flora Argentina. Darwiniana 25: 367-369.

FORTUNATO, R. H. 1986. Revisión del género Bauhinia (Cercideae, Caesalpinioidea, Fabaceae) para la Argentina. Darwiniana 27: 527-557.

FORTUNATO, R. H. \& A. M. CIALDELLA. 1997. Fabaceae. Mimosoideae: Acacia. En: HUNZIKER, A. T (ed.), Fl. Fanerog. Argent. 35: 1-26.

FORTUNATO, R. H. \& E. A. ULIBARRI. 1997. Fabaceae. Cercideae. Detarieae. En: HUNZIKER, A. T. (ed.), Fl. Fanerog. Argent. 33: 1-13.

GAGNON, E., A. BRUNEAU, C. E. HUGHES, L. P. de QUEIROZ \& G. P. LEWIS. 2016. A new generic system for the pantropical Caesalpinia group (Leguminosae). PhytoKeys 71: 1-160. https:// doi.org/10.3897/phytokeys.71.9203

GAMERRO, J. C. \& R. H. FORTUNATO. 2001. Morfología del Polen de las Especies Argentinas de Bauhinia (Cercideae, Caesalpinioideae, Fabaceae). Annals of the Missouri Botanical Garden 88: 145 158. https://doi.org/10.2307/2666135
GÓMEZ-SOSA, E. 2000a. Las especies argentinas de Crotalaria (Leguminosae - Crotalarieae): novedades, descripciones y clave. Gayana Bot. 57: 67-87. https://doi.org/10.4067/S0717-66432000000100006

GÓMEZ-SOSA, E. 2000b. Fabaceae. Millettieae. Robinieae. Indigofereae. En: HUNZIKER, A. T. (ed.), Fl. Fanerog. Argent. 68: 1-30.

GUTIÉRREZ, D. G., L. KATINAS \& S. S. TORRES. 2002. Type material of Carlos L. Spegazzini in the Museo de la Plata Herbarium (LP), Argentina. II: Fabaceae. Darwiniana 40: 77-101.

HOC, P. S. 1981. El género Pithecellobium en la Argentina. Darwiniana 23: 523-558.

HOC, P. S. 1982. El género Pithecellobium (Mimosoideae, Ingeae) en Argentina. II. Estudio del polen. Darwiniana 24: 33-45.

HOC, P. S. 1990. Las especies argentinas de Inga (Leguminosae, Mimosoideae). Darwiniana 30: 237-258.

HOC, P. S. 2005. Fabaceae. Mimosoideae. Ingeae. En: ANTON, A. M. \& F. O. ZULOAGA (eds.), $F l$. Fanerog. Argent. 93: 1-26.

HOC, P. S., R. A. PALACIOS \& M. P. MOM. 2006. Novedades en especies de Vigna e Inga (Leguminosae) para la Argentina. Bol. Soc. Argent. Bot. 41: 331-332.

KRAPOVICKAS, A. \& W. C. GREGORY. 2007. Taxonomía del género Arachis (Leguminosae). Bonplandia (Supl.) 16: 1-205.10. https://doi.org/10.30972/bon.160158

LUCKOW, M. 1993. Monograph of Desmanthus (Leguminosae-Mimosoideae). Systematic Botany Monographs 38: 1-166. https://doi.org/10.2307/25027822

MARTÍNEZ, O. G., M. E. BARRANDEGUY, M. V. GARCÍA, D. A. CACHARANI \& D. E. PRADO. 2013. Presencia de Anadenanthera colubrina var. colubrina (Fabaceae, Mimosiodeae) en Argentina. Darwiniana nueva serie 1: 279-288. https://doi.org/10.14522/darwiniana.2013.12.536

PALACIOS, R. A., P. S. HOC. \& S. I. DREWES. 2001. Fabaceae. Phaseoleae. Phaseolinae: Dolichopsis, Macroptilium, Phaseolus, Vigna. En: HUNZIKER, A. T. (ed.), Fl. Fanerog. Argent. 75: 1-18.

ULIBARRI, E. A. 1996. Sinopsis de Caesalpinia y Hoffmannseggia (Leguminosae-Caesalpinioiedeae) de Sudamérica. Darwiniana 34: 229-348.

ULIBARRI, E. A. 1997. Fabaceae. Caesalpinioideae. En: HUNZIKER, A. T. (ed.), Fl. Fanerog. Argent. 32: $1-26$. 


\section{A. V. Carranza et al. - Fabaceae de Selva Pedemontana de Jujuy}

ULIBARRI, E. A. 2008. Los géneros de Caesalpinioiedeae (Leguminosae) presentes en Sudamérica. Darwiniana 46: 69-163.

VANNI, R. O. 2001. El género Desmodium (Leguminosae, Desmodieae) en Argentina. Darwiniana 39: 255-285.

VANNI, R. O. 2017. The genus Stylosanthes (Fabaceae, Papilionoideae, Dalbergieae) in South America. Bol. Soc. Argent. Bot. 52: 549-585.

https://doi.org/10.31055/1851.2372.v52.n3.18033

VANNI, R. O. \& D. B. KURTZ. 2005. Nueva variedad y precisiones taxonómicas para el género Vicia (Leguminosae) en el Norte de la Argentina. Darwiniana 43: 216-231.

ZALLOCCHI, E. M., A. B. POMILIO \& R. A. PALACIOS. 1992. Estudio quimiotaxonómico de la Subtribu Phaseolinae (PhaseoleaePapilionoideae-Leguminosae) I: Cromatografía de flavonoides de las especies argentinas del género Macroptilium. Darwiniana 31: 299-313.

ZALLOCCHI, E. M., A. B. POMILIO \& R. A. PALACIOS. 1993. Estudio quimiotaxonómico de la Subtribu Phaseolinae (Phaseoleae-PapilionoideaeLeguminosae) II: Cromatografía de flavonoides de las especies argentinas del género Vigna. Darwiniana 32: 139-158.

ZALlOCCHI, E. M., A. B. POMILIO \& R. A. PALACIOS. 1995. Estudio quimiotaxonómico de la Subtribu Phaseolinae (Phaseoleae-PapilionoideaeLeguminosae) III: Flavonoides de las especies argentinas de los géneros Phaseolus y Dolichopsis. Darwiniana 33: 135-148.

ZAPATER, M. A. \& E. C. LOZANO. 2012. Notas morfológicas sobre Bauhinia mollis y Bauhinia argentinensis (Fabaceae, Caesalpinioideae), nuevas citas para Argentina y Bolivia. Darwiniana 50: 339-354. 
Bol. Soc. Argent. Bot. 55 (4) 2020

Tabla 1. Evaluación del estado de conservación de las especies de Fabaceae según UICN (2020) Abreviaturas: EN: En peligro, VU: Vulnerable, NT: Casi amenazada, LC: Preocupación menor, DD: Datos deficientes.

\begin{tabular}{|c|c|c|c|}
\hline Especie & Categoría & Fecha de evaluación & Observaciones \\
\hline Amburana cearensis & EN & enero de 1998 & \\
\hline Anadenanthera colubrina var. cebil & LC & enero de 1998 & \\
\hline Bauhinia forficata & LC & octubre de 2009 & sin especificar variedad \\
\hline Caesalpinia pulcherrima & LC & junio de 2018 & \\
\hline Chloroleucon chacöense & VU & enero de 1998 & \\
\hline Coursetia brachyrhachis & VU & enero de 1998 & \\
\hline Crotalaria pumila & LC & febrero de 2010 & \\
\hline Enterolobium contortisiliquum & LC & junio de 2018 & \\
\hline Erythrina crista-galli & LC & junio de 2018 & sin especificar variedad \\
\hline Erythrina mulungu & LC & junio de 2018 & como E. dominguezii \\
\hline Geoffroea decorticans & LC & agosto de 2010 & sin especificar variedad \\
\hline Inga edulis & LC & noviembre de 2018 & \\
\hline Inga marginata & LC & septiembre de 2010 & \\
\hline Inga saltensis & VU & enero de 1998 & \\
\hline Leptospron adenanthum & LC & marzo de 2017 & como Vigna adenantha \\
\hline Libidibia paraguariensis & VU & enero de 1998 & \\
\hline Macroptilium bracteatum & LC & agosto de 2010 & \\
\hline Mimosa xanthocentra & LC & marzo de 2010 & sin especificar variedad \\
\hline Myroxylon peruiferum & LC & junio de 2018 & \\
\hline Parapiptadenia excelsa & LC & junio de 2018 & \\
\hline Parasenegalia visco & LC & enero de 1998 & como Acacia visco \\
\hline Parkinsonia aculeata & LC & junio de 2018 & \\
\hline Phaseolus lunatus & LC & febrero de 2017 & \\
\hline Poissonia hypoleuca & VU & enero de 1998 & \\
\hline Prosopis alba & NT & enero de 1998 & sin especificar variedad \\
\hline Prosopis nigra & DD & enero de 1998 & \\
\hline Pterogyne nitens & NT & enero de 1998 & \\
\hline Rhynchosia minima & LC & junio de 2010 & \\
\hline Senegalia etilis & VU & enero de 1998 & como Acacia etilis \\
\hline Senegalia praecox & LC & junio de 2018 & \\
\hline Senna obtusifolia & LC & febrero de 2017 & \\
\hline Senna pendula & LC & marzo de 2010 & sin especificar variedad \\
\hline Senna spectabilis & LC & septiembre de 2010 & \\
\hline Sesbania bispinosa & LC & junio de 2016 & \\
\hline Tipuana tipu & LC & junio de 2018 & \\
\hline Vachellia albicorticata & LC & junio de 2018 & \\
\hline Vigna luteola & LC & julio de 2019 & \\
\hline Zapoteca formosa & LC & julio de 2010 & \\
\hline
\end{tabular}

